\title{
$\Xi$ baryon strong decays in a chiral quark model
}

\author{
Li-Ye Xiao and Xian-Hui Zhong * \\ Department of Physics, Hunan Normal University, and Key Laboratory of Low-Dimensional \\ Quantum Structures and Quantum Control of Ministry of Education, Changsha 410081, China
}

\begin{abstract}
The strong decays of $\Xi$ baryons up to $N=2$ shell are studied in a chiral quark model. The strong decay properties of these well-established ground decuplet baryons are reasonably described. We find that (i) $\Xi$ (1690) and $\Xi(1820)$ could be assigned to the spin-parity $J^{P}=1 / 2^{-}$state $\left|70,{ }^{2} 8,1,1, \frac{1}{2}^{-}\right\rangle$and the spin-parity $J^{P}=3 / 2^{-}$ state $\left|70,{ }^{2} 8,1,1, \frac{3}{2}^{-}\right\rangle$, respectively. Slight configuration mixing might exist in these two negative parity states. (ii) $\Xi(1950)$ might correspond to several different $\Xi$ resonances. The broad states $(\Gamma \sim 100 \mathrm{MeV})$ observed in the $\Xi \pi$ channel could be classified as the pure $J^{P}=5 / 2^{-}$octet state $\left|70,{ }^{4} 8,1,1, \frac{5}{2}^{-}\right\rangle$or the mixed state $\left|\Xi \frac{1}{2}^{-}\right\rangle_{3}$ with $J^{P}=1 / 2^{-}$. The $\Xi$ resonances with moderate width $(\Gamma \sim 60 \mathrm{MeV})$ observed in the $\Xi \pi$ channel might correspond to the $J^{P}=1 / 2^{+}$excitation $\left|56,{ }^{4} 10,2,2, \frac{1}{2}^{+}\right\rangle$. The second orbital excitation $\left|56,{ }^{4} 10,2,2, \frac{3}{2}^{+}\right\rangle$and the mixed state $\left|\Xi \frac{1}{2}^{-}\right\rangle_{1}$ might be candidates for the narrow width state observed in the $\Lambda \bar{K}$ channel, however, their spin-parity numbers are incompatible with the moment analysis of the data. (iii) $\Xi(2030)$ could not be assigned to either any spin-parity $J^{P}=7 / 2^{+}$states or any pure $J^{P}=5 / 2^{+}$configurations. It seems to favor the $\left|70,{ }^{2} 8,2,2, \frac{3}{2}^{+}\right\rangle$assignment, however, its spin conflicts with the moment analysis of the data. To find more $\Xi$ resonances, the observations in the $\Xi(1530) \pi$ and $\Sigma(1385) \bar{K}$ channels are necessary.
\end{abstract}

PACS numbers: 12.39.Jh, 13.30.-a, 14.20.Lq, 14.20.Mr

\section{INTRODUCTION}

Understanding the baryon spectrum and the search for the missing baryon resonances are hot topics in hadron physics. For the scarcity of high quality antikaon beams and the small production rate for the $\Xi$ resonances via an electromagnetic probe, the $\Xi$ spectrum is still far from being established. There are only a few data on the $\Xi$ resonances from the old bubble chamber experiments with small statistics. Among the eleven $\Xi$ baryons listed in the review of the Particle Data Group (PDG) [1], only the ground octet and decuplet, $\Xi(1320)$ and $\Xi(1530)$, are well established with four-star ratings. Although the resonances $\Xi(1690), \Xi(1820), \Xi(1950)$ and $\Xi(2030)$ are three-star states in PDG, only $\Xi(1820)$ has a determined spinparity $J^{P}=3 / 2^{-}$.

A few theoretical studies of the $\Xi$ baryon spectrum can be found in the literature [2-14]. All of the phenomenological models can well explain the two ground states $\Xi(1320)$ and $\Xi(1530)$, however, their predictions for the excitations are very different. For example, there is a lot of controversy about the spin-parity of $\Xi(1690)$. It might be the first radial excitation of $\Xi$ with $J^{P}=1 / 2^{+}$according to the mass calculations with a nonrelativistic quark model by Chao $e t$ al. [3]. This classification was also supported by the recent quark model study of Melde et al. [13]. However, with a relativized quark model Capstick and Isgur predicted that the first radial excitation of $\Xi$ should have a larger mass of $\sim 1840 \mathrm{MeV}$ [4]. Recently, Pervin and Roberts suggested that $\Xi(1690)$ could be assigned to the first orbital excitation of $\Xi$ with $J^{P}=1 / 2^{-}$, although their quark model predicted mass is about $35 \mathrm{MeV}$ heavier than the mass of $\Xi(1690)[11]$. They believed that a more microscopic treatment of spin-orbit interactions, can be expected to drive this state to slightly lower

\footnotetext{
*zhongxh@hunnu.edu.cn
}

mass. The calculations from the Skyrme model also indicated that $\Xi(1690)$ should have $J^{P}=1 / 2^{-}[12]$. Furthermore, $\Xi(1690)$ was discussed to be a dynamically generated state with $J^{P}=1 / 2^{-}[15,16]$. It should be mentioned that $B A B A R$ collaboration, in a study of $\Lambda_{c}^{+} \rightarrow \Xi^{-} \pi^{+} K^{+}$, recently found some evidence that $\Xi(1690)$ has $J^{P}=1 / 2^{-}[17]$.

Even for the $\Xi(1820)$ with well determined spin-parity quantum numbers, $J^{P}=3 / 2^{-}$, there still exist many puzzles in the explanations of its nature. Considering $\Xi(1820)$ as a $J^{P}=3 / 2^{-}$orbital excitation, its mass and decay properties could be well explained in the nonrelativistic quark model [3] and algebraic model [6]. However, considering $\Xi(1820)$ as a $J^{P}=3 / 2^{-}$excitation, the mass of $\Xi(1820)$ is obviously underestimated by the relativized quark model [4] and one-boson exchange model [5]. In the unitary chiral approaches, $\Xi(1820)$ was even suggested to be a dynamically generated state from the $\Delta$ decuplet-pion octet chiral interaction [15, 16].

Although $\Xi(1950)$ is a three-star state listed in PDG, not much can be said about its properties [1]. According to various phenomenological model predictions [3, 7-9, 18, 19], many states with spin-parity quantum numbers, $J^{P}=1 / 2^{ \pm}$, $3 / 2^{ \pm}$and $5 / 2^{ \pm}$, could be candidates for $\Xi(1950)$, because their predicted masses are close to $1950 \mathrm{MeV}$. Alitti et al. suggested that the broad $\Xi$ resonance $(\Gamma=80 \pm 40 \mathrm{MeV})$ with a mass of $M=1930 \pm 20 \mathrm{MeV}$ observed by them could be classified as the pure $J^{P}=5 / 2^{-}$octet state [20]. With this assignment, the mass and total and partial decay widths seemed to be reasonably understood. However, the observations of Biagi et al. [21] do not satisfy this classification. Although they observed a $\Xi$ resonance around $1950 \mathrm{MeV}$ in the $\Lambda \bar{K}$ invariant mass distribution, its favorable spin-parity should be $5 / 2^{+}$or its spin should be greater than $5 / 2$ in the natural spin-parity series $7 / 2^{-}, 9 / 2^{+}$, etc [21]. In fact, there might be more than one $\Xi$ near $1950 \mathrm{MeV}[1]$. Recently, Valderrama, Xie and Nieves proposed the existence of three $\Xi(1950)$ states: one of these states would be part of a spin-parity $1 / 2^{+}$decuplet and the other two probably would belong to the $5 / 2^{+}$and $5 / 2^{-}$ 
octets [22].

About $\Xi(2030)$, not many discussions can be found in the literature. A moment analysis of [23] suggested that the spin of $\Xi(2030)$ should be $J \geq 5 / 2$. According to the $\mathrm{SU}(3)$ symmetry of hadrons, Samios et al. predicted that $\Xi(2030)$ was most likely to be the partner of $N(1680), \Lambda(1820)$ and $\Sigma(1915)$ with $J^{P}=5 / 2^{+}[24]$. The quark model mass calculations indicated that the second $J^{P}=5 / 2^{+}$state and $J^{P}=7 / 2^{+}$state might be candidates for $\Xi(2030)[3,11]$. However, the recent strong decay analysis of [22] did not support the classification of [24].

Fortunately, the situation of the poor knowledge about these hyperon resonances is to be changed completely with the running of the Japan Proton Accelerator Research Complex (JPARC) facility, where high quality antikaon beams will be available very soon [25]. Then, the $\Xi$ baryons can be directly produced on the nucleons by the process $\bar{K} N \rightarrow \Xi K$ with large production cross sections. Furthermore, the investigation of $\Xi$ baryons is also one of the major parts of the physics programs at $\overline{\mathrm{P} A N D A}$ via the process $\bar{p} p \rightarrow \Xi \bar{\Xi}$ [26], at Jlab via the photo-production processes, $\gamma p \rightarrow K^{+} K^{+} \Xi^{-}$ and $\gamma p \rightarrow K^{+} K^{+} \Xi^{0} \pi^{-}[27-29]$, and at BESIII via the process Charmonium $\rightarrow \bar{\Xi} \Xi[30,31]$, etc. Thus, these new facilities provide us with good chances to study the $\Xi$ spectrum.

Stimulated by the great progress achieved in experiments, more and more theoretical interests have begun to focus on the $\Xi$ physics. For example, some theoretical studies of the production of $\Xi$ via $\bar{K} N \rightarrow \Xi K[32,33$, and $\gamma p \rightarrow$ $K^{+} K^{+} \Xi^{-}$[34, 35], and the determination of the parity of $\Xi$ resonances in these reactions were started recently [36]. In this work, we make a systematic study of the strong decays of the $\Xi$ resonances in a chiral quark model, which has been developed and successfully used to deal with the strong decays of charmed baryons and heavy-light mesons [37-41]. Here, we mainly focus on the three-star $\Xi$ resonances $\Xi(1690)$, $\Xi(1820), \Xi(1950)$ and $\Xi(2030)$.

This work is organized as follows. In the subsequent section, baryons in the constituent quark model are outlined. Then a brief review of the strong decays described within a chiral quark model approach is given in Sec. III The numerical results are presented and discussed in Sec. IV] Finally, a summary is given in Sec. D

\section{BARYONS IN THE QUARK MODEL}

\section{A. Spatial wave functions}

A baryon containing three quarks may be described by an oscillator potential Hamiltonian [42] in a nonrelativistic form:

$$
\mathcal{H}=\sum_{i=1}^{3} \frac{p_{i}^{2}}{2 m_{i}}+\frac{1}{2} K \sum_{i<j}\left(\mathbf{r}_{j}-\mathbf{r}_{i}\right)^{2}
$$

where $\mathbf{r}_{i}$ and $\mathbf{p}_{i}$ are the coordinate and momentum for the $j$ th quark in the baryon rest frame, respectively. The quarks are confined in an oscillator potential with the potential parameter $K$ independent of the flavor quantum number. One defines the
Jacobi coordinates to eliminate the center mass (c.m.) variables:

$$
\begin{aligned}
\vec{\rho} & =\frac{1}{\sqrt{2}}\left(\mathbf{r}_{1}-\mathbf{r}_{2}\right), \\
\vec{\lambda} & =\frac{1}{\sqrt{6}}\left(\frac{m_{1} \mathbf{r}_{1}+m_{2} \mathbf{r}_{2}}{m_{1}+m_{2}}-2 \mathbf{r}_{3}\right), \\
\mathbf{R}_{c . m .} & =\frac{m_{1} \mathbf{r}_{1}+m_{2} \mathbf{r}_{2}+m_{3} \mathbf{r}_{3}}{M},
\end{aligned}
$$

where $M \equiv m_{1}+m_{2}+m_{3}$. Using the Jacobi coordinates in Eqs. (2)-(4), the oscillator Hamiltonian (1) is reduced to

$$
\mathcal{H}=\frac{P_{c . m .}^{2}}{2 M}+\frac{1}{2 m_{\rho}} \mathbf{p}_{\rho}^{2}+\frac{1}{2 m_{\lambda}} \mathbf{p}_{\lambda}^{2}+\frac{3}{2} K\left(\rho^{2}+\lambda^{2}\right),
$$

where

$$
\mathbf{p}_{\rho}=m_{\rho} \dot{\vec{\rho}}, \quad \mathbf{p}_{\lambda}=m_{\lambda} \dot{\vec{\lambda}}, \quad \mathbf{P}_{c . m .}=M \dot{\mathbf{R}}_{c . m .}
$$

with

$$
m_{\lambda}=\frac{3\left(m_{1}+m_{2}\right) m_{3}}{2 M}, \quad m_{\rho}=\frac{2 m_{1} m_{2}}{m_{1}+m_{2}} .
$$

For a $\Xi$ baryon, it contains two strange $(s)$ quarks and a light $u / d$ quark. According to Eqs.(2)-(4), the coordinates, $\mathbf{r}_{1}, \mathbf{r}_{2}$ and $\mathbf{r}_{3}$, can be translated into functions of the Jacobi coordinates $\lambda$ and $\rho$, which are given by

$$
\begin{aligned}
& \mathbf{r}_{1}=\mathbf{R}_{c . m .}+\frac{1}{\sqrt{6}} \frac{3 m^{\prime}}{2 m+m^{\prime}} \vec{\lambda}+\frac{1}{\sqrt{2}} \vec{\rho}, \\
& \mathbf{r}_{2}=\mathbf{R}_{c . m .}+\frac{1}{\sqrt{6}} \frac{3 m^{\prime}}{2 m+m^{\prime}} \vec{\lambda}-\frac{1}{\sqrt{2}} \vec{\rho}, \\
& \mathbf{r}_{3}=\mathbf{R}_{c . m .}-\sqrt{\frac{2}{3}} \frac{3 m}{2 m+m^{\prime}} \vec{\lambda} .
\end{aligned}
$$

Then, the momenta $\mathbf{p}_{1}, \mathbf{p}_{2}$ and $\mathbf{p}_{3}$ are given by

$$
\begin{aligned}
& \mathbf{p}_{1}=\frac{m}{M} \mathbf{P}_{c . m .}+\frac{1}{\sqrt{6}} \mathbf{p}_{\lambda}+\frac{1}{\sqrt{2}} \mathbf{p}_{\rho}, \\
& \mathbf{p}_{2}=\frac{m}{M} \mathbf{P}_{c . m .}+\frac{1}{\sqrt{6}} \mathbf{p}_{\lambda}-\frac{1}{\sqrt{2}} \mathbf{p}_{\rho}, \\
& \mathbf{p}_{3}=\frac{m^{\prime}}{M} \mathbf{P}_{c . m .}-\sqrt{\frac{2}{3}} \mathbf{p}_{\lambda},
\end{aligned}
$$

where $m$ and $m^{\prime}$ stand for the masses of strange and $u / d$ quarks, respectively.

The spatial wave function is a product of the $\rho$-oscillator and the $\lambda$-oscillator states. With the standard notation, the principal quantum numbers of the $\rho$-oscillator and $\lambda$-oscillator are $N_{\rho}=\left(2 n_{\rho}+l_{\rho}\right)$ and $N_{\lambda}=\left(2 n_{\lambda}+l_{\lambda}\right)$, and the energy of a state is given by

$$
E_{N}=\left(N_{\rho}+\frac{3}{2}\right) \omega_{\rho}+\left(N_{\lambda}+\frac{3}{2}\right) \omega_{\lambda} .
$$

The total principal quantum number (i.e. shell number) $N$ is defined as

$$
N=N_{\rho}+N_{\lambda}
$$


and the frequencies of the $\rho$-mode and $\lambda$-mode are

$$
\omega_{\lambda}=\left(3 K / m_{\lambda}\right)^{1 / 2}, \omega_{\rho}=\left(3 K / m_{\rho}\right)^{1 / 2} .
$$

In the constituent quark model, a useful oscillator parameter, i.e. the potential strength is defined as

$$
\alpha_{\lambda}=\left(m_{\lambda} \omega_{\lambda}\right)^{1 / 2}=\left(\frac{3 m^{\prime}}{2 m+m^{\prime}}\right)^{1 / 4} \alpha_{\rho}
$$

For a $\Xi$ baryon, the constituent mass of a strange quark is close to that of a $u / d$ quark. Thus, the relation

$$
\alpha_{\lambda} \simeq \alpha_{\rho} \equiv \alpha_{h}
$$

is a good approximation.

The total spatial wave function of the oscillator Hamiltonian can be written as [43, 44]

$$
\Psi\left(\mathbf{r}_{1}, \mathbf{r}_{2}, \mathbf{r}_{3}\right)=\frac{e^{i \mathbf{P}_{c . m} \cdot \mathbf{R}_{c . m .}}}{\sqrt{(2 \pi)^{3}}} \Psi_{N L L_{Z}}^{\sigma}(\vec{\rho}, \vec{\lambda})
$$

where $\Psi_{N L L_{Z}}^{\sigma}(\vec{\rho}, \vec{\lambda})$ is the harmonic oscillator wave function, and the $\sigma=s, \rho, \lambda, a$ stands for the representation of the $S_{3}$ group. The harmonic oscillator wave function is given by [43, 44]

$$
\Psi_{N L L_{Z}}^{\sigma}(\vec{\rho}, \vec{\lambda})=P_{N L L_{z}}^{\sigma} \frac{\alpha_{h}^{3}}{\pi^{3 / 2}} e^{-\alpha_{h}^{2}\left(\vec{\rho}^{2}+\vec{\lambda}^{2}\right) / 2},
$$

where $P_{N L L_{z}}^{\sigma}$ is a polynomial of $\rho$ and $\lambda$. Explicitly, the states within $N \leq 2$ can be obtained [44]:

$$
\begin{aligned}
N=0, \quad L=0, \quad P_{000}^{s} & =1, \\
N=1, \quad L=1, P_{111}^{\rho} & =\alpha_{h} \rho_{+}, \\
P_{111}^{\lambda} & =\alpha_{h} \lambda_{+}, \\
N=2, \quad L=0, \quad P_{200}^{s} & =\frac{\alpha_{h}^{2}}{\sqrt{3}}\left(\vec{\rho}^{2}+\vec{\lambda}^{2}-3 \alpha_{h}^{-2}\right), \\
P_{200}^{\rho} & =\frac{\alpha_{h}^{2}}{\sqrt{3}} 2 \vec{\rho} \cdot \vec{\lambda}, \\
P_{200}^{\lambda} & =\frac{\alpha_{h}^{2}}{\sqrt{3}}\left(\vec{\rho}^{2}-\vec{\lambda}^{2}\right), \\
L=2, & P_{222}^{s}=\frac{1}{2} \alpha_{h}^{2}\left(\rho_{+}^{2}+\lambda_{+}^{2}\right), \\
P_{222}^{\rho} & =\alpha_{h}^{2} \rho_{+} \lambda_{+}, \\
P_{222}^{\lambda} & =\frac{1}{2} \alpha_{h}^{2}\left(\rho_{+}^{2}-\lambda_{+}^{2}\right), \\
L=1, & P_{211}^{a}=\alpha_{h}^{2}\left(\rho_{+} \lambda_{z}-\lambda_{+} \rho_{z}\right) .
\end{aligned}
$$

\section{B. Flavor and spin wave functions}

In the quark model, the SU(3) flavor wave functions of the symmetry decuplet are [44]

$$
\phi^{s}= \begin{cases}u и u, & \Delta^{++} \\ \frac{1}{\sqrt{3}}(u u d+u d u+d u u), & \Delta^{+} \\ \frac{1}{\sqrt{3}}(u u s+u s u+s u u), & \Sigma^{*+} \\ d d d, & \Delta^{-} \\ \frac{1}{\sqrt{3}}(u d d+d u d+d d u), & \Delta^{0} \\ \frac{1}{\sqrt{3}}(d d s+d s d+s d d), & \Sigma^{*-} \\ s s s, & \Omega \\ \frac{1}{\sqrt{3}}(u s s+s u s+s s u), & \Xi^{* 0} \\ \frac{1}{\sqrt{3}}(d s s+s d s+s s d), & \Xi^{*-} \\ \frac{1}{\sqrt{6}}(u d s+s u d+d s u+s d u+d u s+u s d), & \Sigma^{* 0}\end{cases}
$$

the mixed-symmetry octet wave functions are [44]

$$
\phi^{\lambda}= \begin{cases}\frac{1}{\sqrt{6}}(2 u u d-d u u-u d u), & \mathrm{p} \\ \frac{1}{\sqrt{6}}(d u d+u d d-2 d d u), & \mathrm{n} \\ \frac{1}{\sqrt{6}}(2 u u s-s u u-u s u), & \Sigma^{+} \\ \frac{1}{2 \sqrt{3}}(s d u+s u d+u s d+d s u-2 u d s-2 d u s), & \Sigma^{0} \\ \frac{1}{\sqrt{6}}(2 d d s-s d d-d s d), & \Sigma^{-} \\ \frac{1}{2}(s u d+u s d-s d u-d s u), & \Lambda \\ \frac{1}{\sqrt{6}}(s u s+u s s-2 s s u), & \Xi^{0} \\ \frac{1}{\sqrt{6}}(d s s+s d s-2 s s d), & \Xi^{-}\end{cases}
$$

while the mixed-antisymmetric octet wave functions are [44]

$$
\phi^{\rho}= \begin{cases}\frac{1}{\sqrt{2}}(u d u-d u u), & \mathrm{p} \\ \frac{1}{\sqrt{2}}(u d d-d u d), & \mathrm{n} \\ \frac{1}{\sqrt{2}}(u s u-s u u), & \Sigma^{+} \\ \frac{1}{2}(s u d+s d u-u s d-d s u), & \Sigma^{0} \\ \frac{1}{\sqrt{2}}(d s d-s d d), & \Sigma^{-} \\ \frac{1}{2 \sqrt{3}}(u s d+s d u-s u d-d s u-2 d u s+2 u d s), & \Lambda \\ \frac{1}{\sqrt{2}}(u s s-s u s), & \Xi^{0} \\ \frac{1}{\sqrt{2}}(d s s-s d s) . & \Xi^{-}\end{cases}
$$

The total antisymmetric singlet flavor wave function is

$$
\phi^{a}=\frac{1}{\sqrt{6}}(u d s+d s u+s u d-d u s-u s d-s d u) .
$$

In the quark model, the typical SU(2) spin wave functions for the baryons can be adopted [45, 46]:

$$
\begin{aligned}
\chi_{3 / 2}^{s} & =\uparrow \uparrow \uparrow, \quad \chi_{-3 / 2}^{s}=\downarrow \downarrow \downarrow, \\
\chi_{1 / 2}^{s} & =\frac{1}{\sqrt{3}}(\uparrow \uparrow \downarrow+\uparrow \downarrow \uparrow+\downarrow \uparrow \uparrow), \\
\chi_{-1 / 2}^{s} & =\frac{1}{\sqrt{3}}(\uparrow \downarrow \downarrow+\downarrow \downarrow \uparrow+\downarrow \uparrow \downarrow),
\end{aligned}
$$


for the spin-3/2 states with symmetric spin wave functions,

$$
\begin{aligned}
\chi_{1 / 2}^{\rho} & =\frac{1}{\sqrt{2}}(\uparrow \downarrow \uparrow-\downarrow \uparrow \uparrow), \\
\chi_{-1 / 2}^{\rho} & =\frac{1}{\sqrt{2}}(\uparrow \downarrow \downarrow-\downarrow \uparrow \downarrow),
\end{aligned}
$$

for the spin-1/2 states with mixed antisymmetric spin wave functions, and

$$
\begin{aligned}
\chi_{1 / 2}^{\lambda} & =-\frac{1}{\sqrt{6}}(\uparrow \downarrow \uparrow+\downarrow \uparrow \uparrow-2 \uparrow \uparrow \downarrow), \\
\chi_{-1 / 2}^{\lambda} & =+\frac{1}{\sqrt{6}}(\uparrow \downarrow \downarrow+\downarrow \uparrow \downarrow-2 \downarrow \downarrow \uparrow),
\end{aligned}
$$

for the spin-1/2 states with mixed symmetric spin wave functions.

The flavor-spin wave functions are representations of $\mathrm{SU}(6)$, which are denoted by $\left|N_{6},{ }^{2 S+1} N_{3}\right\rangle$, where $N_{6}\left(N_{3}\right)$ represents the $\mathrm{SU}(6)(\mathrm{SU}(3))$ representation and $S$ stands for the total spin of the wave function. The symmetric 56 representation wave functions are [44]

$$
\begin{aligned}
\left|56,{ }^{2} 8\right\rangle^{s} & =\frac{1}{\sqrt{2}}\left(\phi^{\rho} \chi^{\rho}+\phi^{\lambda} \chi^{\lambda}\right), \\
\left|56,{ }^{4} 10\right\rangle^{s} & =\phi^{s} \chi^{s}
\end{aligned}
$$

the antisymmetric $\mathbf{7 0}$ representation wave functions are [44]

$$
\begin{aligned}
\left|70,{ }^{2} 8\right\rangle^{\rho} & =\frac{1}{\sqrt{2}}\left(\phi^{\rho} \chi^{\lambda}+\phi^{\lambda} \chi^{\rho}\right) \\
\left|70,{ }^{4} 8\right\rangle^{\rho} & =\phi^{\rho} \chi^{s} \\
\left|70,{ }^{2} 10\right\rangle^{\rho} & =\phi^{s} \chi^{\rho} \\
\left|70,{ }^{2} 1\right\rangle^{\rho} & =\phi^{\alpha} \chi^{\lambda}
\end{aligned}
$$

while the symmetric $\mathbf{7 0}$ representation wave functions are 44]

$$
\begin{aligned}
\left|70,{ }^{2} 8\right\rangle^{\lambda} & =\frac{1}{\sqrt{2}}\left(\phi^{\rho} \chi^{\rho}-\phi^{\lambda} \chi^{\lambda}\right) \\
\left|70,{ }^{4} 8\right\rangle^{\lambda} & =\phi^{\lambda} \chi^{s} \\
\left|70,{ }^{2} 10\right\rangle^{\lambda} & =\phi^{s} \chi^{\lambda} \\
\left|70,{ }^{2} 1\right\rangle^{\lambda} & =\phi^{\alpha} \chi^{\rho}
\end{aligned}
$$

and the antisymmetric $\mathbf{2 0}$ representation wave functions are 44]

$$
\begin{aligned}
\left|20,{ }^{2} 8\right\rangle^{a} & =\frac{1}{\sqrt{2}}\left(\phi^{\rho} \chi^{\lambda}-\phi^{\lambda} \chi^{\rho}\right), \\
\left|20,{ }^{4} 1\right\rangle^{a} & =\phi^{a} \chi^{s} .
\end{aligned}
$$

\section{Total wave functions}

\begin{tabular}{|c|c|c|c|}
\hline state & $1 \mathrm{~N}$ & J L $S$ & wave function \\
\hline$\left|56,{ }^{2} 8,0,0, \frac{1}{2}^{+}\right\rangle$ & 10 & $\begin{array}{lll}\frac{1}{2} & 0 & \frac{1}{2}\end{array}$ & $\left|56,{ }^{2} 8\right\rangle \Psi_{000}^{s}$ \\
\hline$\left|56,{ }^{4} 10,0,0, \frac{3}{2}^{+}\right\rangle$ & ( & $\begin{array}{lll}\frac{3}{2} & 0 & \frac{3}{2} \\
\end{array}$ & $\left|56,{ }^{4} 10\right\rangle \Psi_{000}^{s}$ \\
\hline$\left|56,{ }^{2} 8,2,0, \frac{1}{2}^{+}\right\rangle$ & 12 & $\begin{array}{lll}\frac{1}{2} & 0 & \frac{1}{2}\end{array}$ & $\left|56,{ }^{2} 8\right\rangle \Psi_{200}^{s}$ \\
\hline$\left|56,{ }^{4} 10,2,0, \frac{3}{2}^{+}\right\rangle$ & 2 & $\begin{array}{lll}\frac{3}{2} & 0 & \frac{3}{2}\end{array}$ & $\left|56,{ }^{4} 10\right\rangle \Psi_{200}^{s}$ \\
\hline$\left|56,{ }^{2} 8,2,2, \frac{3}{2}^{+}\right\rangle$ & 2 & $\frac{5}{2} 2 \frac{1}{2}$ & $\left|56,{ }^{2} 8\right\rangle \Psi_{22 L_{z}}^{s}$ \\
\hline$\left|56,{ }^{2} 8,2,2, \frac{5}{2}^{+}\right\rangle$ & 2 & $\frac{5}{2} \quad 2 \quad \frac{1}{2}$ & \\
\hline$\left|56,{ }^{4} 10,2,2, \frac{1}{2}^{+}\right\rangle$ & 2 & $\frac{7}{2} \quad 2 \quad \frac{3}{2}$ & \\
\hline$\left|56,{ }^{4} 10,2,2, \frac{3}{2}^{+}\right\rangle$ & 2 & $\frac{7}{2} 2 \frac{3}{2}$ & $\left|56,{ }^{4} 10\right\rangle \Psi_{22 L_{z}}^{s}$ \\
\hline$\left|56,{ }^{4} 10,2,2, \frac{5}{2}^{+}\right\rangle$ & 2 & $\frac{7}{2} 2 \frac{3}{2}$ & \\
\hline$\left|56,{ }^{4} 10,2,2, \frac{7}{2}^{+}\right\rangle$ & 2 & $\frac{7}{2} \quad 2 \quad \frac{3}{2}$ & \\
\hline$\left|70,^{2} 8,1,1, \frac{1}{2}^{-}\right\rangle$ & 1 & $\begin{array}{lll}\frac{3}{2} & 1 & \frac{1}{2}\end{array}$ & $\left|70,{ }^{2} 8\right\rangle^{\rho} \Psi_{11 L_{z}}^{\rho}+\left|70,,^{2} 8\right\rangle^{\lambda} \Psi_{11 L_{z}}^{\lambda}$ \\
\hline$\left|70,{ }^{2} 8,1,1, \frac{3}{2}^{-}\right\rangle$ & 1 & $\frac{3}{2} \quad 1 \quad \frac{1}{2}$ & \\
\hline$\left|70,{ }^{4} 8,1,1, \frac{1}{2}^{-}\right\rangle$ & 1 & $\begin{array}{lll}\frac{5}{2} & 1 & \frac{3}{2}\end{array}$ & \\
\hline$\left|70,{ }^{4} 8,1,1, \frac{3}{2}^{-}\right\rangle$ & 1 & $\begin{array}{lll}\frac{5}{2} & 1 & \frac{3}{2}\end{array}$ & $\left|70,{ }^{4} 8\right\rangle^{\rho} \Psi_{11 L_{z}}^{\rho}+\left|70,{ }^{4} 8\right\rangle^{\lambda} \Psi_{11 L_{z}}^{\lambda}$ \\
\hline$\left|70,{ }^{4} 8,1,1, \frac{5}{2}^{-}\right\rangle$ & 1 & $\frac{5}{2} \quad 1 \quad \frac{3}{2}$ & \\
\hline$\left|70,^{2} 10,1,1, \frac{1}{2}^{-}\right\rangle$ & 1 & $\begin{array}{lll}\frac{3}{2} & 1 & \frac{1}{2}\end{array}$ & $\left|70,{ }^{2} 10\right\rangle^{\rho} \Psi_{11 L_{z}}^{\rho}+\left|70,{ }^{2} 10\right\rangle^{\lambda} \Psi_{11 L_{z}}^{\lambda}$ \\
\hline$\left|70,^{2} 10,1,1, \frac{3}{2}^{-}\right\rangle$ & 1 & $\frac{3}{2} \quad 1 \quad \frac{1}{2}$ & \\
\hline$\left|70,{ }^{2} 8,2,0, \frac{1}{2}^{+}\right\rangle$ & 2 & $\begin{array}{lll}\frac{1}{2} & 0 & \frac{1}{2}\end{array}$ & $\left|70,^{2} 8\right\rangle^{\rho} \Psi_{200}^{\rho}+\left|70,^{2} 8\right\rangle^{\lambda} \Psi_{200}^{\lambda}$ \\
\hline$\left|70,{ }^{4} 8,2,0, \frac{3}{2}^{+}\right\rangle$ & 2 & $\begin{array}{lll}\frac{3}{2} & 0 & \frac{3}{2}\end{array}$ & $\left|70,{ }^{4} 8\right\rangle^{\rho} \Psi_{200}^{\rho}+\left|70,{ }^{4} 8\right\rangle^{\lambda} \Psi_{200}^{\lambda}$ \\
\hline$\left|70,^{2} 10,2,0, \frac{1}{2}^{+}\right\rangle$ & 2 & $\begin{array}{lll}\frac{1}{2} & 0 & \frac{1}{2}\end{array}$ & $\left|70,{ }^{2} 10\right\rangle^{\rho} \Psi_{200}^{\rho}+\left|70,^{2} 10\right\rangle^{\lambda} \Psi_{200}^{\lambda}$ \\
\hline$\left|70,^{2} 8,2,2, \frac{3}{2}^{+}\right\rangle$ & 2 & $\begin{array}{lll}\frac{5}{2} & 2 & \frac{1}{2}\end{array}$ & $\left|70,{ }^{2} 8\right\rangle^{\rho} \Psi_{22 L_{z}}^{\rho}+\left|70,{ }^{2} 8\right\rangle^{\lambda} \Psi_{22 L_{z}}^{\lambda}$ \\
\hline$\left|70,{ }^{2} 8,2,2, \frac{5}{2}^{+}\right\rangle$ & 2 & $\frac{5}{2} \quad 2 \quad \frac{1}{2}$ & \\
\hline$\left|70,{ }^{4} 8,2,2, \frac{1}{2}^{+}\right\rangle$ & & $\frac{7}{2} \quad 2 \quad \frac{3}{2}$ & \\
\hline$\left|70,{ }^{4} 8,2,2, \frac{3}{2}^{+}\right\rangle$ & 2 & $\frac{7}{2} \quad 2 \quad \frac{3}{2}$ & $\left|70,{ }^{4} 8\right\rangle^{\rho} \Psi_{22 L_{z}}^{\rho}+\left|70,{ }^{4} 8\right\rangle^{\lambda} \Psi_{22 L_{z}}^{\lambda}$ \\
\hline$\left|70,{ }^{4} 8,2,2, \frac{5}{2}^{+}\right\rangle$ & 2 & $\frac{7}{2} 2 \frac{3}{2}$ & \\
\hline$\left|70,{ }^{4} 8,2,2, \frac{7}{2}^{+}\right\rangle$ & 2 & $\frac{7}{2} \quad 2 \quad \frac{3}{2}$ & \\
\hline$\left|70,,^{2} 10,2,2, \frac{3}{2}^{+}\right\rangle$ & 2 & $\frac{5}{2} \quad 2 \quad \frac{1}{2}$ & $\left|70,{ }^{2} 10\right\rangle^{\rho} \Psi_{22 L_{z}}^{\rho}+\left|70,{ }^{2} 10\right\rangle^{\lambda} \Psi_{22 L_{z}}^{\lambda}$ \\
\hline$\left|70,^{2} 10,2,2, \frac{5}{2}^{+}\right\rangle$ & 2 & $\frac{5}{2} \quad 2 \quad \frac{1}{2}$ & \\
\hline
\end{tabular}

The total spin-flavor-space wave functions are the $\mathrm{SU}(6) \otimes$ $\mathrm{O}(3)$ representations, which are denoted by

$$
|S U(6) \otimes O(3)\rangle=\left|N_{6},{ }^{2 S+1} N_{3}, N, L, J^{P}\right\rangle .
$$

The spin-flavor-space wave functions have to be permutationsymmetric because the baryon color wave function is totally anti-symmetric. The spin-flavor-space wave functions up to $N=2$ shell are listed in Tab. I. So far no experimental evidence for the existence of the $20 \mathrm{SU}(6)$ representation has been found, thus, we do not consider these excitations in this work.

TABLE I: The spin-flavor-space wave functions of different light baryons, denoted by $\left|\mathbf{N}_{6},{ }^{2 S+1} \mathbf{N}_{3}, N, L, J^{P}\right\rangle$. The ClebschGordan series for the spin and angular-momentum addition $\left|J, J_{z}\right\rangle=$ $\sum_{L_{z}+S_{z}=J_{z}}\left\langle L L_{z}, S S_{z} \mid J J_{z}\right\rangle \Psi_{N L L_{z}}^{\sigma} \chi_{S_{z}}$ has been omitted.

\section{STRONG DECAY OF A BARYON IN THE CHIRAL QUARK MODEL}

In the chiral quark model, the effective low energy quarkmeson pseudoscalar coupling at tree level is given by [47-50]

$$
H_{m}=\sum_{j} \frac{1}{f_{m}} \bar{\psi}_{j} \gamma_{\mu}^{j} \gamma_{5}^{j} \psi_{j} \vec{\tau} \cdot \partial^{\mu} \vec{\phi}_{m},
$$

where $\psi_{j}$ represents the $j$ th quark field in a baryon, and $f_{m}$ is the meson's decay constant. The pseudoscalar meson octet $\phi_{m}$ 
is expressed as

$$
\phi_{m}=\left(\begin{array}{ccc}
\frac{1}{\sqrt{2}} \pi^{0}+\frac{1}{\sqrt{6}} \eta & \pi^{+} & K^{+} \\
\pi^{-} & -\frac{1}{\sqrt{2}} \pi^{0}+\frac{1}{\sqrt{6}} \eta & K^{0} \\
K^{-} & \bar{K}^{0} & -\sqrt{\frac{2}{3}} \eta
\end{array}\right) .
$$

To match the nonrelativistic harmonic oscillator spatial wave function ${ }^{N} \Psi_{L L_{z}}$ in the quark model, we adopt the nonrelativistic form of Eq. (51) in the calculations, which is given by [47-50]

$$
\begin{aligned}
H_{m}^{n r} \simeq & \sum_{j=1}^{3}\left\{\frac{\omega_{m}}{E_{f}+M_{f}} \boldsymbol{\sigma}_{j} \cdot \mathbf{P}_{f}+\frac{\omega_{m}}{E_{i}+M_{i}} \boldsymbol{\sigma}_{j} \cdot \mathbf{P}_{i}\right. \\
& \left.-\boldsymbol{\sigma}_{j} \cdot \mathbf{q}+\frac{\omega_{m}}{2 \mu_{q}} \boldsymbol{\sigma}_{j} \cdot \mathbf{p}_{j}^{\prime}\right\} I_{j} \varphi_{m},
\end{aligned}
$$

where $\sigma_{j}$ is the Pauli spin operator on the the $j$ th quark of the baryon, and $\mu_{q}$ is a reduced mass given by $1 / \mu_{q}=1 / m_{j}+1 / m_{j}^{\prime}$ with $m_{j}$ and $m_{j}^{\prime}$ for the masses of the $j$ th quark in the initial and final baryons, respectively. For emitting a meson, we have $\varphi_{m}=e^{-i \mathbf{q} \cdot \mathbf{r}_{j}}$, and for absorbing a meson we have $\varphi_{m}=e^{i \mathbf{q} \cdot \mathbf{r}_{j}}$. In the above nonrelativistic expansion, $\mathbf{p}_{j}^{\prime}\left(=\mathbf{p}_{j}-m_{j} / M \mathbf{P}_{c . m .}\right)$ is the internal momentum operator for the $j$ th quark in the baryon rest frame. $\omega_{m}$ and $\mathbf{q}$ are the energy and three-vector momentum of the meson, respectively. $\mathbf{P}_{i}$ and $\mathbf{P}_{f}$ stand for the momenta of the initial final baryons, respectively. The isospin operator $I_{j}$ in Eq. (53) is expressed as

$$
I_{j}= \begin{cases}a_{j}^{\dagger}(u) a_{j}(s) & \text { for } K^{+}, \\ a_{j}^{\dagger}(s) a_{j}(u) & \text { for } K^{-}, \\ a_{j}^{\dagger}(d) a_{j}(s) & \text { for } K^{0}, \\ a_{j}^{\dagger}(s) a_{j}(d) & \text { for } \bar{K}^{0}, \\ a_{j}^{\dagger}(u) a_{j}(d) & \text { for } \pi^{-}, \\ a_{j}^{\dagger}(d) a_{j}(u) & \text { for } \pi^{+}, \\ \frac{1}{\sqrt{2}}\left[a_{j}^{\dagger}(u) a_{j}(u)-a_{j}^{\dagger}(d) a_{j}(d)\right] & \text { for } \pi^{0}, \\ \frac{1}{\sqrt{2}}\left[a_{j}^{\dagger}(u) a_{j}(u)+a_{j}^{\dagger}(d) a_{j}(d)\right] \cos \phi_{P} & \\ -a_{j}^{\dagger}(s) a_{j}(s) \sin \phi_{P} & \text { for } \eta,\end{cases}
$$

where $a_{j}^{\dagger}(u, d, s)$ and $a_{j}(u, d, s)$ are the creation and annihilation operators for the $u, d$ and $s$ quarks, and $\phi_{P}$ is the mixing angle of $\eta$ meson in the flavor basis [1, 51].

In the calculations, we select the initial-baryon-rest system for the decay processes. The energies and momenta of the initial baryons are denoted by $\left(E_{i}, \mathbf{P}_{i}\right)$, while those of the final state mesons and baryons are denoted by $\left(\omega_{f}, \mathbf{q}\right)$ and $\left(E_{f}, \mathbf{P}_{f}\right)$, respectively. Note that $\mathbf{P}_{i}=0$ and $\mathbf{P}_{f}=-\mathbf{q}$. For a light pseudoscalar meson emission in the strong decay process of a baryon, $\mathcal{B} \rightarrow \mathcal{B}^{\prime} \mathrm{M}(\mathbf{q})$, the partial decay amplitudes can be worked out according to

$$
\mathcal{M}\left[\mathcal{B} \rightarrow \mathcal{B}^{\prime} \mathbb{M}(\mathbf{q})\right]=3\left\langle\mathcal{B}^{\prime}\left|\left\{G \sigma_{3} \cdot \mathbf{q}+h \sigma_{3} \cdot \mathbf{p}_{3}^{\prime}\right\} I_{3} e^{-i \mathbf{q} \cdot \mathbf{r}_{3}}\right| \mathcal{B}\right\rangle(55)
$$

with

$$
G \equiv-\frac{\omega_{m}}{E_{f}+M_{f}}-1, h \equiv \frac{\omega_{m}}{2 \mu_{q}},
$$

where $\mathcal{B}$ and $\mathcal{B}^{\prime}$ stand for the initial and final baryon wave functions listed in Tab. I]

With the derived decay amplitudes, one can calculate the width by [37]

$$
\Gamma=\left(\frac{\delta}{f_{m}}\right)^{2} \frac{\left(E_{f}+M_{f}\right)|q|}{4 \pi M_{i}} \frac{1}{2 J_{i}+1} \sum_{J_{i z} J_{f z}}\left|\mathcal{M}_{I_{i z}, J_{f z}}\right|,
$$

where the $J_{i z}$ and $J_{f z}$ stand for the the total angular momenta of the initial and final baryons, respectively. $\delta$ as a global parameter accounts for the strength of the quark-meson couplings.

In the calculation, the standard quark model parameters are adopted. Namely, we set $m_{u}=m_{d}=350 \mathrm{MeV}, m_{s}=450$ $\mathrm{MeV}$, for the constituent quark masses. The harmonic oscillator parameter $\alpha_{h}$ in the wave function ${ }^{N} \Psi_{L L_{z}}$ is taken as $\alpha_{h}=0.40 \mathrm{GeV}$. The decay constants for $\pi, K$ and $\eta$ mesons are taken as $f_{\pi}=132 \mathrm{MeV}, f_{K}=f_{\eta}=160 \mathrm{MeV}$, respectively. The masses of the mesons and baryons used in the calculations are adopted from the Particle Data Group [1].

\section{RESULTS AND ANALYSIS}

TABLE II: The partial and total decay widths $(\mathrm{MeV})$ of the wellestablished baryons $\Xi(1530), \Sigma(1385)$ and $\Delta(1232)$, which correspond to the same representation $\left|56,{ }^{4} 10,0,0, \frac{3}{2}^{+}\right\rangle$. The experimental data are obtained from PDG.

\begin{tabular}{|c|c|c|c|c|}
\hline \hline state & channel & $\Gamma_{i}^{\text {th }}$ & $\Gamma_{\text {total }}^{\text {th }}$ & $\Gamma_{\text {total }}^{\text {exp }}$ \\
\hline$\Xi(1530)^{0}$ & $\Xi^{-} \pi^{+}$ & 5.6 & 9.1 (input) & $9.1 \pm 0.5$ \\
& $\Xi^{0} \pi^{0}$ & 3.5 & & \\
\hline$\Xi(1530)^{-}$ & $\Xi^{0} \pi^{-}$ & 7.1 & 10.3 & $9.9_{-1.9}^{+1.7}$ \\
& $\Xi^{-} \pi^{0}$ & 3.2 & & \\
\hline$\Sigma(1385)^{+}$ & $\Sigma^{+} \pi^{0}$ & 1.9 & 27.1 & $36 \pm 0.7$ \\
& $\Sigma^{0} \pi^{+}$ & 1.5 & & \\
& $\Lambda^{0} \pi^{+}$ & 23.7 & & \\
\hline$\Sigma(1385)^{0}$ & $\Sigma^{+} \pi^{-}$ & 1.8 & 27.9 & $36 \pm 5$ \\
& $\Sigma^{-} \pi^{+}$ & 1.3 & & \\
& $\Lambda^{0} \pi^{0}$ & 24.8 & & \\
\hline$\Sigma(1385)^{-}$ & $\Sigma^{-} \pi^{0}$ & 1.7 & 28.7 & $39.4 \pm 2.1$ \\
& $\Sigma^{0} \pi^{-}$ & 1.8 & & \\
& $\Lambda^{0} \pi^{-}$ & 25.2 & & \\
\hline$\Delta(1232)^{++}$ & $p \pi^{+}$ & 63.9 & 63.9 & $114 \sim 120$ \\
\hline$\Delta(1232)^{+}$ & $p \pi^{0}$ & 43.7 & 64.7 & $114 \sim 120$ \\
& $n \pi^{+}$ & 21.0 & & \\
\hline$\Delta(1232)^{0}$ & $p \pi^{-}$ & 21.3 & 64.3 & $114 \sim 120$ \\
& $n \pi^{0}$ & 43.0 & & \\
\hline
\end{tabular}

\section{A. $\Xi(1530)$}

$\Xi(1530)$ is the only $\Xi$ resonance whose properties are all reasonably well known. According to the classification of 
the quark model, it is assigned to the $\left|56,{ }^{4} 10,0,0, \frac{3}{2}^{+}\right\rangle$representation. In the present work, the measured width for $\Xi(1530)^{0} \rightarrow \Xi \pi$ as an input (i.e. $\Gamma=9.1 \mathrm{MeV}$ [1]) to determine parameter $\delta$ in Eq. 57), which gives

$$
\delta=0.576 .
$$

With this determined parameter, we can predict the other $\Xi$ resonance strong decays. First, the strong decays of $\Xi(1530)^{-}$ are calculated. The results have been listed in Tab. [I where we find that our predictions are in agreement with the data. Furthermore, we have noted that $\Sigma(1385)$ and $\Delta(1232)$ also correspond to the $\left|56,{ }^{4} 10,0,0, \frac{3}{2}^{+}\right\rangle$representation. They are the SU(3)-flavor counterparts of $\Xi(1530)$. Applying the determined value for $\delta$, we calculate the strong decays of the $\Sigma(1385)$ and $\Delta(1232)$ resonances, the results are listed in Tab. [1]as well.

It is seen that the predicted widths for $\Sigma(1385)$ are compatible with the experimental data, and the partial decay width ratio

$$
\frac{\Gamma[\Sigma(1385) \rightarrow \Sigma \pi]}{\Gamma[\Sigma(1385) \rightarrow \Lambda \pi]} \simeq 0.134 \pm 0.09,
$$

are in good agreement with the PDG value [1].

The predicted widths for $\Delta(1232)$ are underestimated by about a factor of 2 compared with the data. In fact, there are systemic underestimations of the decay widths for the $\Delta$ and nucleon excitations as well. The reason is that the parameters used in this work are only fine tuned for the $\Xi$ resonances to make more reliable predictions for the their decay properties. For simplicity, we do not carry out a global fit to strange and non-strange baryons. We have noted that the global parameter $\delta=0.576$ used for the $\Xi$ resonances in this work is slightly smaller than that used for the $\Delta$ and nucleon resonances in [52].

As a whole, the predicted strong decay properties for $\Xi(1530)^{-}, \Sigma(1385)$ are compatible with the experimental data. If a global fit to strange and non-strange baryons is carried out, the predicted strong decay width of $\Delta(1232)$ can be more close to the data. Thus, the SU(3)-flavor symmetry approximately holds in these ground decuplet baryons.

\section{B. $\Xi(1690)$}

$\Xi(1690)$ is a three-star state listed in PDG. Its decay width might be less than $30 \mathrm{MeV}$ [1]. Three decay modes $\Lambda \bar{K}$, $\Sigma \bar{K}$ and $\Xi \pi$ were observed in experiments. Recently, $B A B A R$ Collaboration found some evidence that the $\Xi^{0}(1690)$ has $J^{P}=1 / 2^{-}$in the study of $\Lambda_{c}^{+} \rightarrow \Xi^{-} \pi^{+} K^{+}$[17].

If $\Xi(1690)$ is a $J^{P}=1 / 2^{-}$resonance, it should correspond to one of the first orbital excitation states: $\left|70,{ }^{2} 8,1,1, \frac{1}{2}^{-}\right\rangle$, $\left|70,{ }^{4} 8,1,1, \frac{1}{2}^{-}\right\rangle$and $\left|70,{ }^{2} 10,1,1, \frac{1}{2}^{-}\right\rangle$or a mixed state between them. Considering $\Xi(1690)$ as the $\left|70,{ }^{2} 8,1,1, \frac{1}{2}^{-}\right\rangle$, $\left|70,{ }^{4} 8,1,1, \frac{1}{2}^{-}\right\rangle$and $\left|70,{ }^{2} 10,1,1, \frac{1}{2}^{-}\right\rangle$, respectively, we calculate their decay properties, which are listed in Tab. III Comparing the predictions with the measurements, we find that if
$\Xi(1690)$ is assigned to $\left|70,,^{2} 8,1,1, \frac{1}{2}^{-}\right\rangle$, the calculated decay width

$$
\Gamma \approx 48 \mathrm{MeV},
$$

and partial decay width ratios

$$
\frac{\Gamma(\Xi \pi)}{\Gamma(\Sigma \bar{K})} \simeq 0.2, \frac{\Gamma(\Sigma \bar{K})}{\Gamma(\Lambda \bar{K})} \approx 1.0, \frac{\Gamma(\Xi(1530) \pi)}{\Gamma(\Sigma \bar{K})} \approx 0.0002,
$$

are roughly in agreement with the measurements. Neither $\left|70,{ }^{4} 8,1,1, \frac{1}{2}^{-}\right\rangle$nor $\left|70,{ }^{2} 10,1,1, \frac{1}{2}^{-}\right\rangle$could be considered as an assignment to $\Xi(1690)$ because their partial decay width ratios disagree with the observations.

TABLE III: The total and partial decay widths (MeV) of $\Xi(1690)$ with different $J^{P}=1 / 2^{-}$assignments.

\begin{tabular}{|c|r|r|r|r|r|}
\hline \hline assignment & $\Xi \pi$ & $\Sigma \bar{K}$ & $\Lambda \bar{K}$ & $\Xi(1530) \pi$ & $\Gamma_{\text {total }}$ \\
\hline$\left|70,{ }^{2} 8,1,1, \frac{1}{2}^{-}\right\rangle$ & 3.69 & 22.30 & 22.15 & 0.005 & 48.14 \\
\hline$\left|70,{ }^{4} 8,1,1, \frac{1}{2}^{-}\right\rangle$ & 59.05 & 5.58 & 22.15 & 0.001 & 86.78 \\
\hline$\left|70,{ }^{2} 10,1,1, \frac{1}{2}^{-}\right\rangle$ & 3.69 & 1.39 & 5.54 & 0.005 & 10.63 \\
\hline
\end{tabular}

As we know, configuration mixing between several states with the same $J^{P}$ often occurs via some interactions. Thus, $\Xi(1690)$ might be a mixed state between $\left|70,{ }^{2} 8,1,1, \frac{1}{2}^{-}\right\rangle$, $\left|70,{ }^{4} 8,1,1, \frac{1}{2}^{-}\right\rangle$and $\left|70,{ }^{2} 10,1,1, \frac{1}{2}^{-}\right\rangle$. Now, we consider the physical states with $J^{P}=\frac{1}{2}^{-}$as mixed states between $\left|70,{ }^{2} 8\right\rangle$, $\left|70,{ }^{4} 8\right\rangle$ and $\left|70,{ }^{2} 10\right\rangle$. According to the standard CKM matrix method, the physical states can be express as

$$
\left(\begin{array}{l}
\left|\Xi \frac{1}{2}^{-}\right\rangle_{1} \\
\left|\Xi \frac{1}{2}^{-}\right\rangle_{2} \\
\left|\Xi \frac{1}{2}^{-}\right\rangle_{3}
\end{array}\right)=U\left(\begin{array}{c}
\left|70,{ }^{2} 8\right\rangle \\
\left|70,{ }^{4} 8\right\rangle \\
\left|70,{ }^{2} 10\right\rangle
\end{array}\right)
$$

with

$$
U=\left(\begin{array}{ccc}
c_{12} c_{13} & s_{12} c_{13} & s_{13} \\
-s_{12} c_{23}-c_{12} s_{23} s_{13} & c_{12} c_{23}-s_{12} s_{23} s_{13} & s_{23} c_{13} \\
s_{12} s_{23}-c_{12} c_{23} s_{13} & -c_{12} s_{23}-s_{12} c_{23} s_{13} & c_{23} c_{13}
\end{array}\right),
$$

where $c_{i j} \equiv \cos \theta_{i j}$ and $s_{i j} \equiv \sin \theta_{i j}$ with $\theta_{i j}$ the mixing angles to be determined by the experimental data.

In present work, we take $\Xi(1690)$ as $\left|\Xi \frac{1}{2}^{-}\right\rangle_{2}$. By fitting the experimental data of DIONISI 78 [53] (see Tab. IV], we have obtained $\theta_{12} \simeq 5^{0}, \theta_{13} \simeq 105^{\circ}$ and $\theta_{23} \simeq 95^{\circ}$. The theoretical predictions compared with the data were listed in Tab.IV From the table, we find that the decay properties of $\Xi(1690)$ could be well described with these determined mixing angles. Thus, $\Xi(1690)$ could be a mixed state.

With these determined mixing angles, Eq. 62 can be explicitly written as

$$
\left(\begin{array}{c}
\left|\Xi \frac{1}{2}^{-}\right\rangle_{1} \\
\left|\Xi(1690) \frac{1}{2}^{-}\right\rangle \\
\left|\Xi \frac{1}{2}^{-}\right\rangle_{3}
\end{array}\right)=\left(\begin{array}{ccc}
-0.26 & -0.02 & 0.97 \\
-0.95 & -0.17 & -0.26 \\
0.17 & -0.98 & 0.02
\end{array}\right)\left(\begin{array}{c}
\left|70,{ }^{2} 8\right\rangle \\
\left|70,{ }^{4} 8\right\rangle \\
\left|70,{ }^{2} 10\right\rangle
\end{array}\right),
$$


TABLE IV: The predicted total and partial decay widths (MeV) and partial decay width ratios (MeV) of $\Xi(1690) \equiv \Psi_{2}$ compared with the experiment data of DIONISI 78 [53]. The mixing angles are $\theta_{12}=5^{0}$, $\theta_{13}=75^{\circ}$ and $\theta_{23}=95^{\circ}$.

\begin{tabular}{|c|c|c|c|c|c|c|}
\hline \hline channel & $\Gamma_{i}^{\text {th }}$ & $\Gamma_{\text {total }}^{\text {th }}$ & $\Gamma_{\text {total }}^{\text {exp }}$ & channel ratio & $R_{\text {th }}$ & $R_{\text {exp }}$ \\
\hline$\Xi \pi$ & 1.0 & 37 & $44_{-23}^{+23}$ & $\Gamma(\Sigma \bar{K}) / \Gamma(\Lambda \bar{K})$ & 2.88 & $2.7_{-0.9}^{+0.9}$ \\
$\Sigma \bar{K}$ & 27.0 & & & $\Gamma(\Xi \pi) / \Gamma(\Sigma \bar{K})$ & 0.04 & $<0.09$ \\
$\Lambda \bar{K}$ & 9.4 & & & $\Gamma(\Xi(1530) \pi) / \Gamma(\Sigma \bar{K})$ & $\sim 10^{-4}$ & $<0.06$ \\
$\Xi(1530) \pi$ & 0.01 & & & & & \\
\hline \hline
\end{tabular}

From the above equation, it is obviously seen that the main component of the physical state $\Xi(1690)$ is $\left|70,{ }^{2} 8,1,1,1 / 2^{-}\right\rangle$ $(\sim 90 \%)$, which slightly mixes with $\left|70,{ }^{2} 10,1,1,1 / 2^{-}\right\rangle(\sim$ $7 \%)$ and $\left|70,{ }^{4} 8,1,1,1 / 2^{-}\right\rangle(\sim 3 \%)$.

In Ref. [54, the $B A B A R$ Collaboration studied the spin of $\Xi(1690)$ from $\Lambda_{c}^{+} \rightarrow \Lambda K^{+} \bar{K}^{0}$. They found that the spin of $\Xi(1690)$ is consistent with the value $J=1 / 2$. However, they could not determine the parity of $\Xi(1690)$. According to the mass predictions of quark model, the mass of the first radial $(2 S)$ excitation of $\Xi$ with $J^{P}=\frac{1}{2}^{+}$might be close to $1690 \mathrm{MeV}$ [3, 13]. Thus, we should consider the possibility of $\Xi(1690)$ as the positive parity radial $(2 S)$ excitations with $J^{P}=\frac{1}{2}^{+}$. In the constituent quark model, there are three radial $(2 S)$ excitations with $J^{P}=\frac{1}{2}^{+}:\left|56,{ }^{2} 8,2,0, \frac{1}{2}^{+}\right\rangle$, $\left|70,{ }^{2} 8,2,0, \frac{1}{2}^{+}\right\rangle$and $\left|70,{ }^{2} 10,2,0, \frac{1}{2}^{+}\right\rangle$. In our calculation, those states' total decay widths are too small to compare with the experimental data. The predicted partial decay width ratios are incompatible with the observations as well. Thus, these states with $J^{P}=\frac{1}{2}^{+}$as assignments to $\Xi(1690)$ should be excluded. Furthermore, we also study the strong decay properties of the other first orbital excitation states with $J^{P}=3 / 2^{-}$ and $J^{P}=5 / 2^{-}$. Their decay properties are very different from those of $\Xi(1690)$. For simplicity, the calculated results are not shown in this work.

In a word, $\Xi(1690)$ is most likely to be the first orbital excitation of $\Xi$ with $J^{P}=1 / 2^{-}$. There might exist configuration mixing in $\Xi(1690)$. The main component of $\Xi(1690)$ is $\left|70,{ }^{2} 8,1,1,1 / 2^{-}\right\rangle$, which slightly mixes with $\left|70,{ }^{2} 10,1,1,1 / 2^{-}\right\rangle$and $\left|70,{ }^{4} 8,1,1,1 / 2^{-}\right\rangle$. Our predictions are consistent with the experimental observations. The recent mass calculations of a constituent quark model support the classification of $\Xi(1690)$ as a $J^{P}=\frac{1}{2}^{-}$octet resonance [11]. The calculations of a Skyrme model [12] and unitary chiral approaches [15, 16] also indicated that $\Xi(1690)$ has $J^{P}=\frac{1}{2}^{-}$.

Since $\Xi(1690)$ corresponds to the physical state $\left|\Xi \frac{1}{2}^{-}\right\rangle_{2}$ in Eq. 64), as the counterparts of $\Xi(1690)$, the other two physical states, $\left|\Xi \frac{1}{2}^{-}\right\rangle_{1}=-0.26\left|70,{ }^{2} 8\right\rangle-0.02\left|70,{ }^{4} 8\right\rangle+0.97\left|70,{ }^{2} 10\right\rangle$ and $\left|\Xi \frac{1}{2}^{-}\right\rangle_{3}=0.17\left|70,{ }^{2} 8\right\rangle-0.98\left|70,{ }^{4} 8\right\rangle+0.02\left|70,{ }^{2} 10\right\rangle$, might be observed in experiments. It is easily seen that the main component of $\left|\Xi \frac{1}{2}^{-}\right\rangle_{1}$ is $\left|70,,^{2} 10,1,1,1 / 2^{-}\right\rangle$( 94\%), while $\left|\Xi \frac{1}{2}^{-}\right\rangle_{3}$ is dominated by $\left|70,{ }^{4} 8,1,1,1 / 2^{-}\right\rangle(\sim 96 \%)$. According to the analysis in large $N_{c}$ QCD [7, 8] and quark model [2], the masses of $\left|\Xi \frac{1}{2}^{-}\right\rangle_{1}$ and $\left|\Xi \frac{1}{2}^{-}\right\rangle_{3}$ are around $1920 \mathrm{MeV}$. The strong decay properties of $\left|\Xi \frac{1}{2}^{-}\right\rangle_{1}$ and $\left|\Xi \frac{1}{2}^{-}\right\rangle_{3}$ are studied as well. Considering the uncertainties of the mass predictions, we vary the masses of $\left|\Xi \frac{3}{2}^{-}\right\rangle_{1}$ and $\left|\Xi \frac{3}{2}^{-}\right\rangle_{3}$ from $1860 \mathrm{MeV}$ to $1980 \mathrm{MeV}$. The results are shown in Fig. 1. From the figure, we find that $\left|\Xi \frac{1}{2}^{-}\right\rangle_{1}$ is a narrow state with a width of $\Gamma \sim 25$ $\mathrm{MeV}$, while $\left|\Xi \frac{1}{2}^{-}\right\rangle_{3}$ is a broad state with a width of $\sim 100 \mathrm{MeV}$. The main decay channels of $\left|\Xi \frac{1}{2}^{-}\right\rangle_{1}$ are $\Sigma \bar{K}$ and $\Lambda \bar{K}$, while the decays of $\left|\Xi \frac{1}{2}^{-}\right\rangle_{3}$ are governed by $\Xi \pi$ and $\Lambda \bar{K}$.

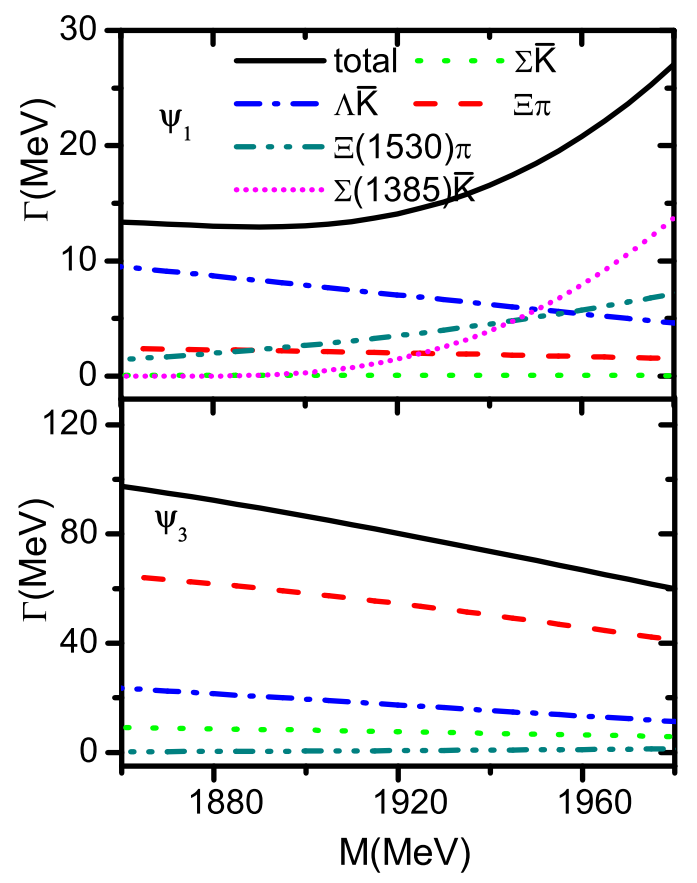

FIG. 1: The decay properties of $\left|\Xi \frac{1}{2}^{-}\right\rangle_{1}$ and $\left|\Xi \frac{1}{2}^{-}\right\rangle_{3}$, respectively, where $\Psi_{1} \equiv\left|\Xi \frac{1}{2}^{-}\right\rangle_{1}$ and $\Psi_{3} \equiv\left|\Xi \frac{1}{2}^{-}\right\rangle_{3}$.

\section{C. $\Xi(1820)$}

In 1987, Biagi et al. measured the spin-parity of $\Xi(1820)$ [21]. They found that its spin-parity is consistent with $J=3 / 2^{-}$, which is in good agreement with the quark model predictions [3, 11, 13]. In the present work, the study of the well-established resonance $\Xi(1820)$, on the one hand, can provide an important test of our model; on the other hand, it can let us obtain more information about the nature of $\Xi(1820)$.

Assigning $\Xi(1820)$ to the negative states with $J^{P}=1 / 2^{-}$, $3 / 2^{-}$and $5 / 2^{-}$, respectively, their strong decays are calculated with our model. The results are listed in Tab. $\mathrm{V}$, where we find that only the $\left|70,{ }^{2} 8,1,1, \frac{3}{2}^{-}\right\rangle$can be assigned to $\Xi(1820)$. The detail comparisons of theoretical predictions with measurements are shown in Tab. VI, from which it is seen that both the decay width and the partial decay ratios are in agreement with the measurements of ALITTI 69 [55]. Our predictions 
about the spin-parity values of $\Xi(1820)$ are consistent with the other model predictions and experimental determinations.

TABLE V: The decay widths $(\mathrm{MeV})$ of $\Xi(1820)^{0}$ with different assignments.

\begin{tabular}{|c|c|c|c|c|c|}
\hline \hline assignment & $\Xi \pi$ & $\Sigma \bar{K}$ & $\Lambda \bar{K}$ & $\Xi(1530) \pi$ & $\Gamma_{\text {total }}$ \\
\hline$\left|70,{ }^{2} 8,1,1, \frac{3}{2}^{-}\right\rangle$ & 2.85 & 10.63 & 7.25 & 11.98 & 32.71 \\
\hline$\left|70,{ }^{4} 8,1,1, \frac{1}{2}^{-}\right\rangle$ & 64.80 & 23.94 & 20.56 & 0.36 & 109.66 \\
$\left|70,{ }^{4} 8,1,1, \frac{3}{2}^{-}\right\rangle$ & 4.56 & 0.26 & 0.72 & 11.95 & 17.49 \\
$\left|70,{ }^{4} 8,1,1, \frac{5}{2}^{-}\right\rangle$ & 27.40 & 1.59 & 4.35 & 1.51 & 34.85 \\
\hline$\left|70,{ }^{2} 10,1,1, \frac{1}{2}^{-}\right\rangle$ & 4.05 & 5.98 & 5.14 & 1.43 & 16.60 \\
$\left|70,{ }^{2} 10,1,1, \frac{3}{2}^{-}\right\rangle$ & 2.85 & 0.66 & 1.81 & 11.98 & 17.31 \\
\hline
\end{tabular}

TABLE VI: The predicted total and partial decay width widths $(\mathrm{MeV})$ and partial decay width ratios of $\Xi(1820)$ as the $\left|70,{ }^{2} 8,1,1, \frac{3}{2}^{-}\right\rangle$assignment compared with the experimental data of ALITTI 69 [55].

\begin{tabular}{|c|c|c|c|c|c|}
\hline \hline channel & $\Gamma_{i}^{\text {th }}$ & $\Gamma_{\text {total }}^{\text {th }}$ & $\Gamma_{\text {total }}^{\text {exp }}$ & $\left.\frac{\Gamma_{i}}{\Gamma_{\text {total }}}\right|_{\text {th }}$ & $\left.\frac{\Gamma_{i}}{\Gamma_{\text {total }}}\right|_{\exp }$ \\
\hline$\Xi \pi$ & 2.9 & 32.8 & $24_{-10}^{+15}$ & 0.09 & $0.1 \pm 0.1$ \\
$\Sigma \bar{K}$ & 10.6 & & & 0.32 & $0.30 \pm 0.15$ \\
$\Lambda \bar{K}$ & 7.3 & & & 0.22 & $0.30 \pm 0.15$ \\
$\Xi(1530) \pi$ & 12.0 & & & 0.37 & $0.30 \pm 0.15$ \\
\hline
\end{tabular}

Although by considering $\Xi(1820)$ as a pure state $\left|70,{ }^{2} 8,1,1, \frac{3}{2}^{-}\right\rangle$, we note that the theoretical predictions are in good agreement with the experimental observations, there still exists room for configuration mixing in $\Xi$ (1820) for the uncertainties of the experimental data. Chao et al. employed a quark model to study the mass spectrum of $\Xi$ resonances [3]. According to their study, $\Xi(1820)$ should be a mixed state, which dominates by the octet $\left|70,{ }^{2} 8,1,1, \frac{3}{2}^{-}\right\rangle$ components $(\sim 90 \%)$, while contains small components of $\left|70,{ }^{2} 10,1,1, \frac{3}{2}^{-}\right\rangle(\sim 9 \%)$ and $\left|70,{ }^{4} 8,1,1, \frac{3}{2}^{-}\right\rangle(\sim 1 \%)$. Thus, we consider the physical state $\Xi(1820)$ as an admixture between the octet and decuplet states with $J^{P}=\frac{3}{2}^{-}$. Using the CKM matrix method discussed in IVB and fitting the observed strong decay properties, we obtain

$$
\left(\begin{array}{c}
\left|\Xi \frac{3}{2}^{-}\right\rangle_{1} \\
\left|\Xi(1820) \frac{3}{2}^{-}\right\rangle \\
\left|\Xi \frac{3}{2}^{-}\right\rangle_{3}
\end{array}\right)=\left(\begin{array}{ccc}
-0.08 & -0.98 & 0.17 \\
0.96 & -0.13 & -0.26 \\
0.27 & 0.14 & 0.95
\end{array}\right)\left(\begin{array}{c}
\left|70,{ }^{2} 8\right\rangle \\
\left|70,{ }^{4} 8\right\rangle \\
\left|70,{ }^{2} 10\right\rangle
\end{array}\right) .
$$

The theoretical results compared with the data are listed in Tab.VII where we find that the predicted partial decay width ratios are in agreement with the experiment data of ALITTI 69 [55] as well. Considering configuration mixing effects in $\Xi(1820)$, the decay width is closer to the center values of observations. From the mixing parameters obtained in Eq. 65), it is seen that the main component of $\Xi(1820)$ is $\left|70,{ }^{2} 8,1,1, \frac{3}{2}^{-}\right\rangle$, which is about $92 \%$. As a mixed state, $\Xi(1820)$ also contains small components of $\left|70,{ }^{4} 8,1,1, \frac{3}{2}^{-}\right\rangle$ $(\sim 0.02 \%)$ and $\left|70,{ }^{2} 10,1,1, \frac{3}{2}^{-}\right\rangle(\sim 0.07 \%)$. Our results are compatible with the predictions in the quark model [3], and the large $N_{c}$ QCD approach [7, 8]. It should be mentioned that $\Xi(1820)$ was also suggested to be a dynamically generated state with $J^{P}=3 / 2^{-}[15,16]$.

TABLE VII: The predicted total and partial decay widths $(\mathrm{MeV})$ and partial decay width ratios of $\Xi(1820)$ as the mixed state $\left|\Xi(1820) \frac{3}{2}^{-}\right\rangle$ with mixing angles $\theta_{12}=85^{\circ}, \theta_{13}=170^{\circ}$ and $\theta_{23}=165^{\circ}$. For a comparison, the experimental data of ALITTI 69 [55] are listed as well.

\begin{tabular}{|c|c|c|c|c|c|}
\hline \hline channel & $\Gamma_{i}^{\text {th }}$ & $\Gamma_{\text {total }}^{\text {th }}$ & $\Gamma_{\text {total }}^{\exp }$ & $\left.\frac{\Gamma_{i}}{\Gamma_{\text {total }}}\right|_{\text {th }}$ & $\left.\frac{\Gamma_{i}}{\Gamma_{\text {total }}}\right|_{\exp }$ \\
\hline$\Xi \pi$ & 2.1 & 23.3 & $24_{-10}^{+15}$ & 0.09 & $0.1 \pm 0.1$ \\
$\Sigma \bar{K}$ & 8.0 & & & 0.34 & $0.30 \pm 0.15$ \\
$\Lambda \bar{K}$ & 9.2 & & & 0.40 & $0.30 \pm 0.15$ \\
$\Xi(1530) \pi$ & 4.0 & & & 0.17 & $0.30 \pm 0.15$ \\
\hline
\end{tabular}

In brief, $\Xi(1820)$ could be approximately taken as a pure $\left|70,{ }^{2} 8,1,1, \frac{3}{2}^{-}\right\rangle$state. Slight configuration mixing may exist in it. All the experimental observations of $\Xi(1820)$ could be well understood in the constituent quark model.

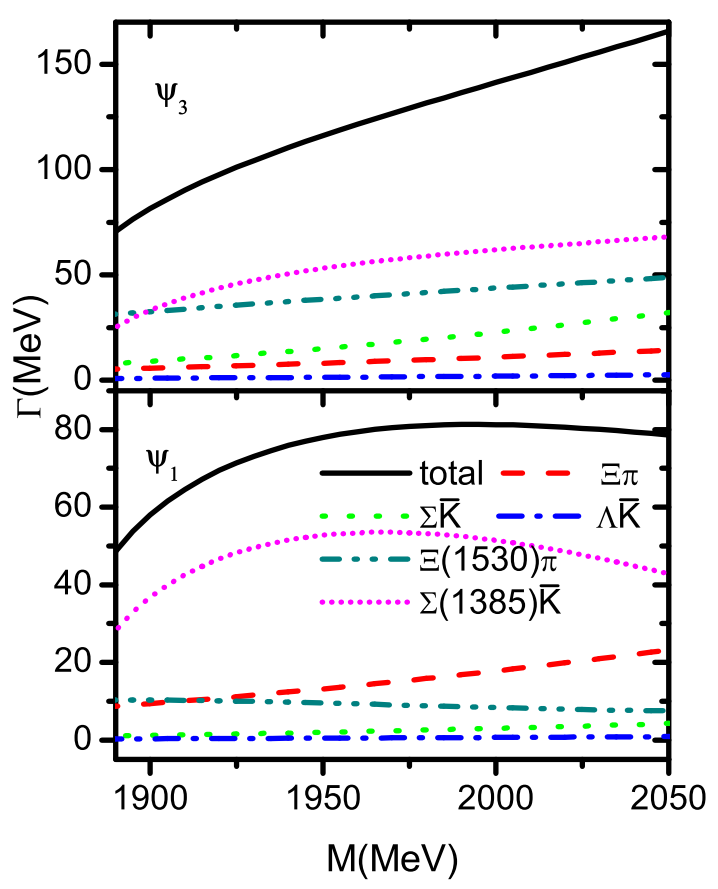

FIG. 2: The decay properties of $\left|\Xi \frac{3}{2}^{-}\right\rangle_{1}$ and $\left|\Xi \frac{3}{2}^{-}\right\rangle_{3}$, respectively, where $\Psi_{1} \equiv\left|\Xi \frac{3}{2}^{-}\right\rangle_{1}$ and $\Psi_{3} \equiv\left|\Xi \frac{3}{2}^{-}\right\rangle_{3}$.

If $\Xi(1820)$ is a mixed state indeed, its counterparts $\left|\Xi \frac{3}{2}^{-}\right\rangle_{1}$ and $\left|\Xi \frac{3}{2}^{-}\right\rangle_{3}$ in Eq. 65 might be observed in experiments as well. From Eq. 65] it is seen that the main components of $\left|\Xi \frac{3}{2}^{-}\right\rangle_{1}$ and $\left|\Xi \frac{3}{2}^{-}\right\rangle_{3}$ are $\left|70,{ }^{4} 8,1,1,3 / 2^{-}\right\rangle(\sim 96 \%)$ and $\left|70,{ }^{2} 10,1,1,3 / 2^{-}\right\rangle(\sim 90 \%)$, respectively. According to the quark model predictions [2, 3], the masses of $\left|\Xi \frac{3}{2}^{-}\right\rangle_{1}$ and $\left|\Xi \frac{3}{2}^{-}\right\rangle_{3}$ are $\sim 1910 \mathrm{MeV}$ and $\sim 1970 \mathrm{MeV}$, respectively. The later large QCD calculations [7, 8] gave similar predictions 
to those of quark models. The strong decay properties of $\left|\Xi \frac{3}{2}^{-}\right\rangle_{1}$ and $\left|\Xi \frac{3}{2}^{-}\right\rangle_{3}$ are studied as well. The results are shown in Fig. 2. For the uncertainties of the mass predictions, we vary the masses of $\left|\Xi \frac{3}{2}^{-}\right\rangle_{1}$ and $\left|\Xi \frac{3}{2}^{-}\right\rangle_{3}$ from $1850 \mathrm{MeV}$ to $2050 \mathrm{MeV}$. From the calculations, we find that if the masses of these two states are larger than the threshold of $\Sigma(1385) \bar{K}$, the decay channel $\Sigma(1385) \bar{K}$ dominates their decays. Furthermore, $\Xi(1530) \pi$ contributes significantly to the strong decays of these two states.

Finally, it should be pointed out that although the predicted masses of $\left|\Xi \frac{3}{2}^{-}\right\rangle_{1}$ and $\left|\Xi \frac{3}{2}^{-}\right\rangle_{3}$ are close to that of $\Xi(1950)$, the decay modes and partial decay width ratios are not consistent with the observations. Thus, $\Xi(1950)$ does not favor any $J^{P}=$ $3 / 2^{-}$assignments.

\section{D. $\Xi(1950)$}

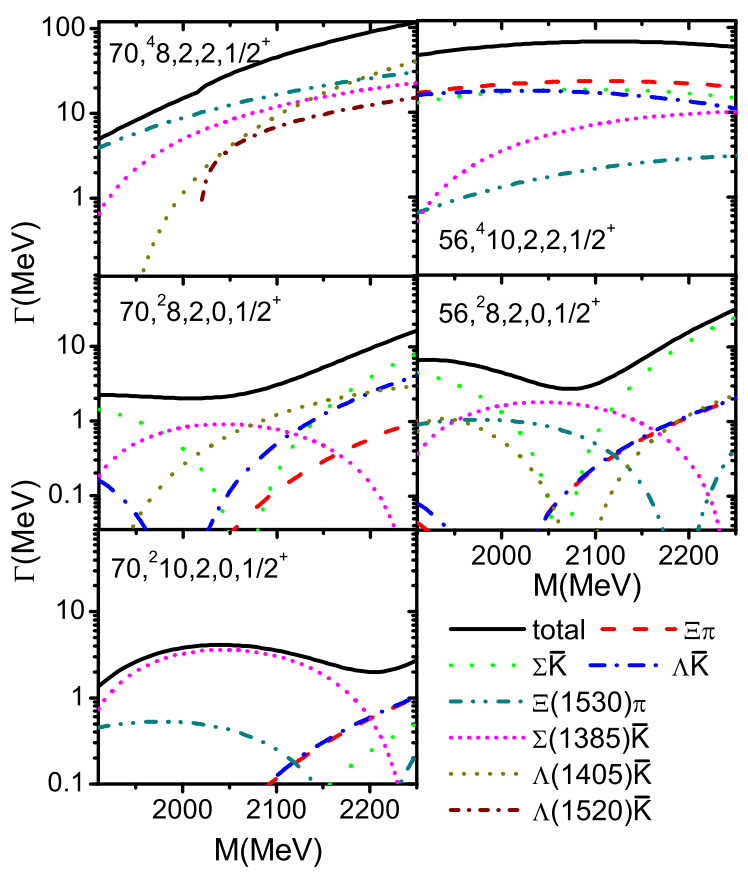

FIG. 3: Strong decay properties of the $J^{P}=1 / 2^{+}$excitations in the $N=2$ shell.

$\Xi(1950)$ was first observed by Badier et al. in the invariant mass distribution $\Xi \pi$ of $K^{-} p \rightarrow \Xi^{-} K \pi$ process [57], its observed mass and width are $1933 \pm 16 \mathrm{MeV}$ and $\Gamma \simeq 140 \pm 35$ $\mathrm{MeV}$, respectively. Later, several experimental groups also found structures with a mass of $\sim 1950 \pm 50 \mathrm{MeV}$ in the other processes. $\Xi(1950)$ was only observed in $\Xi \pi, \Xi(1530) \pi$ and $\Lambda \bar{K}$ decay channels [1]. Most of these structures were observed in the $\Xi \pi$ channel. In the $\Lambda \bar{K}$ channel, only Biagi et al. claimed that they observed one narrow structure $(\Gamma \simeq 25 \pm 15$ $\mathrm{MeV}$ ) with a mass of $M \simeq 1963 \mathrm{MeV}$ [21]. They estimated

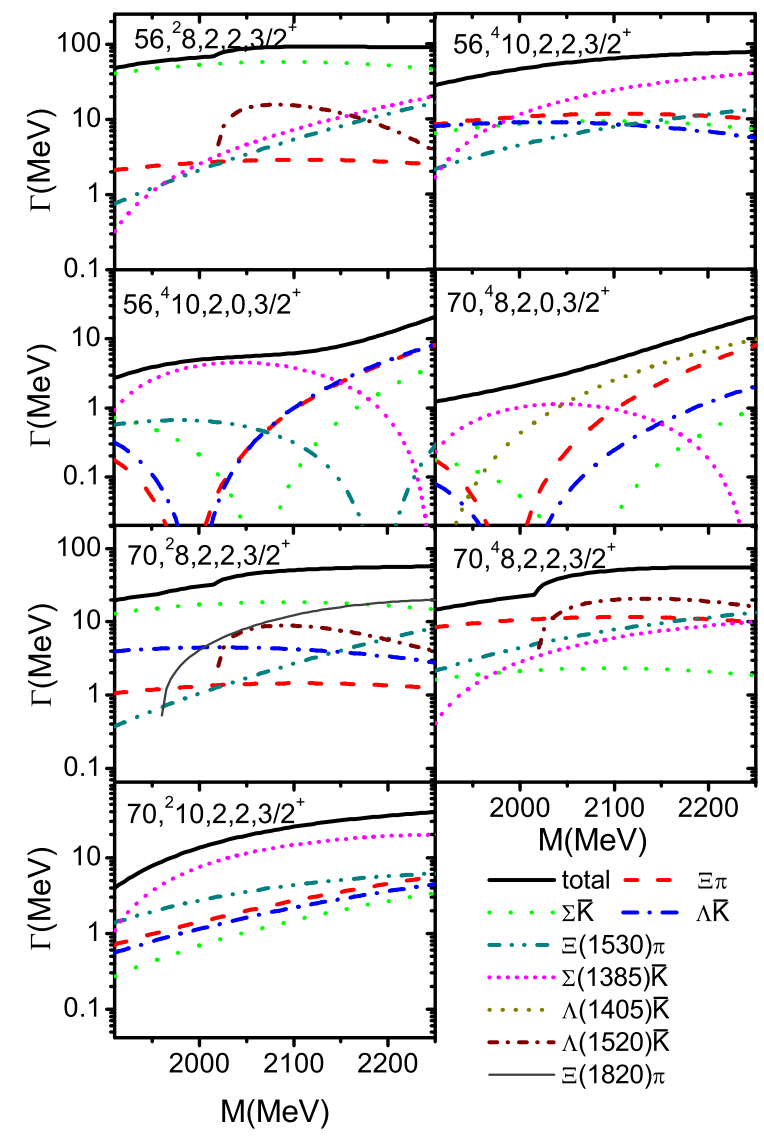

FIG. 4: Strong decay properties of the $J^{P}=3 / 2^{+}$excitations in the $N=2$ shell.

an upper limit on the ratio of partial widths $\Gamma(\Sigma \bar{K}) / \Gamma(\Lambda \bar{K})$ of 2.3, and also suggested that the spin-parity of this resonance should be $5 / 2^{+}$or its spin should be greater than $5 / 2$ in the natural spin-parity series $7 / 2^{-}, 9 / 2^{+}$, etc. While in the $\Xi(1530) \pi$ channel, only Briefel et al. reported that they observed a broad structure $(\Gamma \simeq 60 \pm 39 \mathrm{MeV})$ with a mass of $M \simeq 1964 \mathrm{MeV}$ [58]. Although $\Xi(1950)$ is a three-star $\Xi$ resonance listed in PDG, not much can be said about its properties. According to various model predictions, there are several $\Xi$ resonances in the $1900-2000 \mathrm{MeV}$ region [3, 7-9].

$$
\text { 1. } J^{P}=5 / 2^{-} \text {assignment }
$$

$\Xi(1950)$ was first classified as the pure octet $\Xi$ resonance with $J^{P}=5 / 2^{-}$by Alitti et al. [20]. In 1968, they observed a $\Xi$ resonance whose mass and width are $M=1930 \pm 20$ $\mathrm{MeV}$ and $\Gamma=80 \pm 40 \mathrm{MeV}$, respectively. The resonance parameters were very close to the observations of Badier et al. in 1965 [57]. The Gell-Mann-Okubo mass formula indicates this state might be the pure $J^{P}=5 / 2^{-}$octet $\Xi$ resonance [24, 59]. The detailed SU(3) study of the total and partial decay widths 


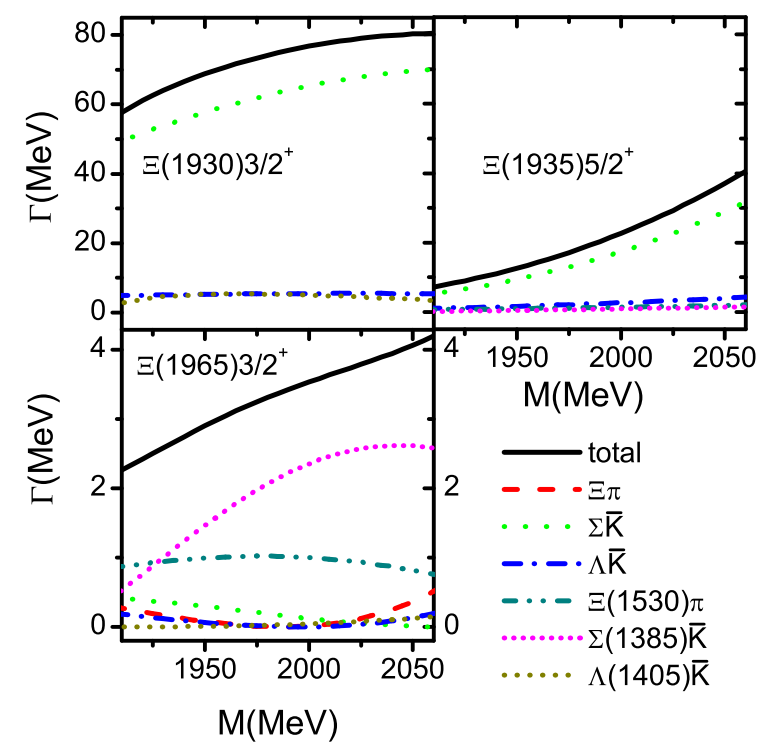

FIG. 5: Strong decay properties of $J^{P}=3 / 2^{+}$and $J^{P}=5 / 2^{+}$mixed states suggested by Chao et al. [3].

of the $J^{P}=5 / 2^{-}$octet baryons seemed to give a reasonable and consistent picture [20]. This classification was also supported by some studies of the mass spectrum of $\Xi$ resonances in various quark models [3, 11].

With $\Xi(1950)$ as an assignment to the $J^{P}=5 / 2^{-}$octet $\Xi$ resonance, we study its strong decay properties, the results are listed in Tab.VIII. It is seen that the $J^{P}=5 / 2^{-}$assignment is a broad state with a width of $\sim 100 \mathrm{MeV}$. Its strong decays are dominated by the $\Xi \pi$ channel. The partial decay widths of $\Xi(1530) \pi$ and $\Lambda \bar{K}$ are sizeable. Our predictions are in compatible with those in [20]. Thus, the broad $\Xi$ resonances observed in $\Xi \pi$ channel might be good candidates for the $J^{P}=5 / 2^{-}$ octet state.

As a by-product, we calculate the strong decays of the other members of $J^{P}=5 / 2^{-}$octet baryons, $N(1675) \frac{5}{2}^{-}, \Sigma(1775) \frac{5}{2}^{-}$ and $\Lambda(1830) \frac{5}{2}^{-}$. The results are listed in Tab. VIII as well. From the table, it is found that our predictions of the strong decay properties of $N(1675) \frac{5}{2}^{-}, \Sigma(1775) \frac{5}{2}^{-}$and $\Lambda(1830) \frac{5}{2}^{-}$ are in reasonable agreement with the observations.

\section{2. $J^{P}=1 / 2^{-}$assignment}

Recently, Valderrama, Xie and Nieves proposed the existence of a spin-parity state $J^{P}=1 / 2^{-}$decuplet belonging to $\Xi(1950)$ [22]. Now we discuss the possibilities of $\Xi(1950)$ as an assignment to the $J^{P}=1 / 2^{-}$states. In Sec. IV B, we predicted $\Xi(1690)$ is a mixed state with $J^{P}=1 / 2^{-}$. According to the mass calculations of constituent quark models [3, 14] and Large $N_{c}$ QCD [7, 8], the masses of the counterparts of $\Xi(1690),\left|\Xi \frac{1}{2}^{-}\right\rangle_{1}$ and $\left|\Xi \frac{1}{2}^{-}\right\rangle_{3}$, might be close to $1950 \mathrm{MeV}$.

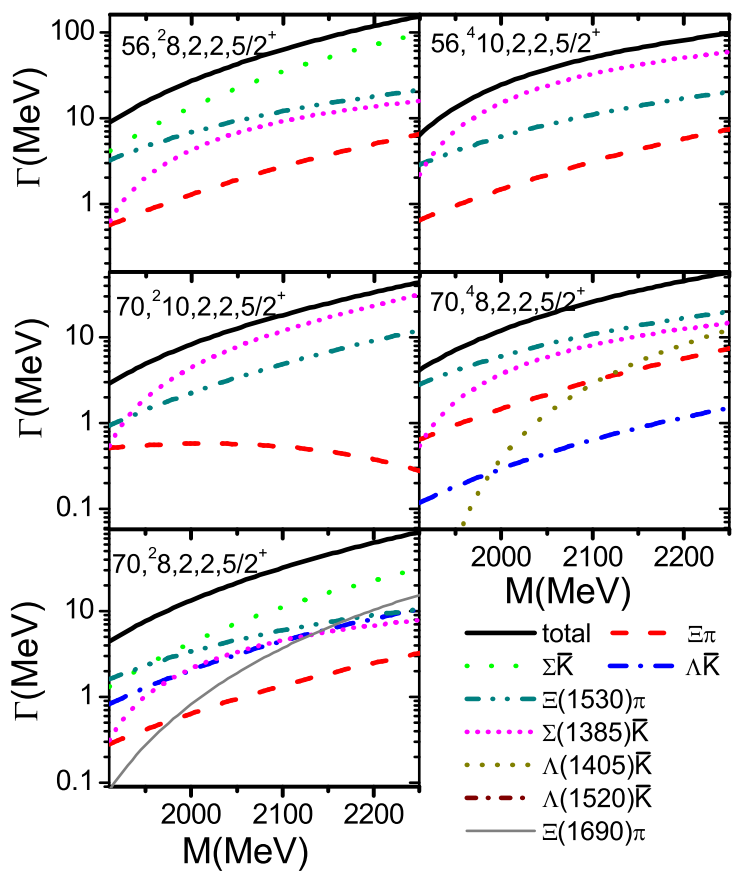

FIG. 6: Strong decay properties of the $J^{P}=5 / 2^{+}$excitations in the $N=2$ shell.

Thus, they might be candidates for $\Xi(1950)$. The strong decay properties of $\left|\Xi \frac{1}{2}^{-}\right\rangle_{1}$ and $\left|\Xi \frac{1}{2}^{-}\right\rangle_{3}$ had been studied in Sec. IV B (see Fig. (1). Considering $\left|\Xi \frac{1}{2}^{-}\right\rangle_{1}$ as an assignment to $\Xi(1950)$, both the decay width and partial width ratio,

$$
\Gamma \simeq 27 \mathrm{MeV}, \frac{\Gamma(\Sigma \bar{K})}{\Gamma(\Lambda \bar{K})} \simeq 2.0,
$$

are in agreement with the observations of Biagi 87C [21]; however, the spin-parity $J^{P}=1 / 2^{-}$disagrees with their suggestion. While assigning $\left|\Xi \frac{1}{2}^{-}\right\rangle_{3}$ to $\Xi(1950)$, we note that its width and partial width ratios

$$
\Gamma \simeq 84 \mathrm{MeV}, \frac{\Gamma(\Sigma \bar{K})}{\Gamma(\Lambda \bar{K})} \simeq 1.6, \frac{\Gamma(\Xi \pi)}{\Gamma(\Sigma \bar{K})} \simeq 2.3,
$$

are consistent with those of the broad structures observed in the $\Xi \pi$ channel. Thus, the spin-parity $1 / 2^{-}$mixed state $\left|\Xi \frac{1}{2}^{-}\right\rangle_{3}$ could be a good assignment to $\Xi(1950)$.

\section{3. $J^{P}=1 / 2^{+}$assignment}

In Ref. [12], Oh predicted that $\Xi(1950)$ might have $J^{P}=$ $1 / 2^{+}$in the Skyrme model. We calculate the strong decays of all the excitations of $\Xi$ with $J^{P}=1 / 2^{+}$in the $N=2$ shell. The results are shown in Fig. 3. From the figure, it is found that if the second orbital excitation $\left|56,{ }^{4} 10,2,2, \frac{1}{2}^{+}\right\rangle$is considered as 


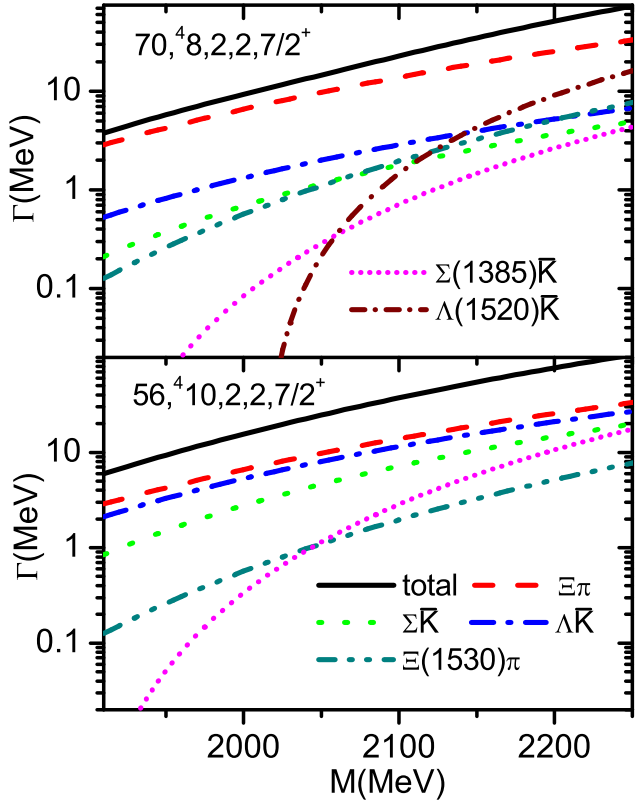

FIG. 7: Strong decay properties of the $J^{P}=7 / 2^{+}$excitations in the $N=2$ shell.

TABLE VIII: The total and partial decay widths $(\mathrm{MeV})$ of the wellestablished four-star baryons $N(1675), \Sigma(1775)$ and $\Lambda(1830)$, which correspond to $\left|70,{ }^{4} 8,1,1, \frac{5}{2}^{-}\right\rangle$. The data are obtained from PDG.

\begin{tabular}{|l|c|c|c|c|c|c|}
\hline \hline assignment & channel & $\Gamma_{i}^{\text {th }}$ & $\Gamma_{\text {total }}^{\text {th }}$ & $\Gamma_{\text {total }}^{\text {exp }}$ & $\left.\frac{\Gamma_{i}}{\Gamma_{\text {total }}}\right|_{\text {th }}$ & $\left.\frac{\Gamma_{i}}{\Gamma_{\text {total }}}\right|_{\text {exp }}$ \\
\hline$N(1675) \frac{5}{2}^{-}$ & $n \pi$ & 25.3 & 81 & $130 \sim 165$ & 0.31 & $0.35-0.45$ \\
& $\Delta \pi$ & 50.6 & & & 0.62 & $0.50-0.60$ \\
& $N \eta$ & 5.8 & & & 0.07 & 0.01 \\
\hline$\Sigma(1775) \frac{5}{2}^{-}$ & $\Sigma \pi$ & 8.0 & 76 & $105 \sim 135$ & 0.11 & $0.02-0.05$ \\
& $\Lambda \pi^{0}$ & 19.1 & & & 0.25 & $0.14-0.20$ \\
& $n \bar{K}$ & 32.1 & & & 0.42 & $0.37-0.43$ \\
& $\Sigma(1385) \pi$ & 4.8 & & & 0.06 & $0.08-0.12$ \\
& $\Delta(1232) \bar{K}$ & 1.0 & & & 0.01 & $\ldots$ \\
& $\Lambda(1520) \pi$ & 11.2 & & & 0.14 & $0.17-0.23$ \\
\hline$\Lambda(1830) \frac{5}{2}^{-}$ & $\Sigma \pi$ & 52.2 & 94 & $60 \sim 110$ & 0.55 & $0.35-0.75$ \\
& $\Sigma(1385) \pi$ & 41.7 & & & 0.45 & $>0.15$ \\
\hline$\Xi(1950) \frac{5}{2}^{-}$ & $\Xi \pi$ & 71.33 & 105 & $80 \pm 40$ & 0.68 & $\ldots$ \\
& $\Sigma \bar{K}$ & 7.8 & & & 0.07 & $\ldots$ \\
& $\Lambda \bar{K}$ & 13.6 & & & 0.13 & $\ldots$ \\
& $\Xi(1530) \pi$ & 10.9 & & & 0.10 & $\ldots$ \\
& $\Sigma(1385) \bar{K}$ & 1.2 & & & 0.01 & $\ldots$ \\
\hline
\end{tabular}

an assignment to $\Xi(1950)$, the decay width and partial width ratios are

$$
\Gamma \simeq 53 \mathrm{MeV}, \frac{\Gamma(\Sigma \bar{K})}{\Gamma(\Lambda \bar{K})} \simeq 0.88, \frac{\Gamma(\Xi \pi)}{\Gamma(\Sigma \bar{K})} \simeq 1.27
$$

The decay width and decay modes are consistent with the observations of Goldwasser et al. [60]. It should be pointed out that the predicted mass of various models for $\left|56,{ }^{4} 10,2,2, \frac{1}{2}^{+}\right\rangle$ is $\sim 2 \mathrm{GeV}$ [3, 9, 14], which is slightly larger than the observation.

$$
\text { 4. } J^{P}=3 / 2^{+} \text {assignment }
$$

Furthermore, we calculate the strong decays of all the excitations of $\Xi$ with $J^{P}=3 / 2^{+}$in the $N=2$ shell. The results are shown in Fig. 4 . It is found that the second orbital excitation $\left|56,{ }^{4} 10,2,2, \frac{3}{2}^{+}\right\rangle$might be a candidate for $\Xi(1950)$. The decay width

$$
\Gamma \simeq 36 \mathrm{MeV}
$$

and the partial width ratios

$$
\frac{\Gamma(\Sigma \bar{K})}{\Gamma(\Lambda \bar{K})} \simeq 0.88, \frac{\Gamma(\Xi \pi)}{\Gamma(\Sigma \bar{K})} \simeq 1.27, \frac{\Gamma(\Xi \pi)}{\Gamma(\Xi(1530) \pi)} \simeq 3.16
$$

are in agreement with the observations of Biagi 87C [21] and APSELL 70 [56]. However, the spin-parity of $\left|56,{ }^{4} 10,2,2, \frac{3}{2}^{+}\right\rangle$is not consistent with the moment analysis of Biagi 87C [21]. Furthermore, the predicted mass of $\left|56,{ }^{4} 10,2,2, \frac{3}{2}^{+}\right\rangle$in large $N_{c}$ QCD is obviously larger than that of $\Xi(1950)[9]$.

Including configuration mixing effects, Chao et al. predicted two $J^{P}=3 / 2^{+}$mixed states with masses of $M=1930$ and $1965 \mathrm{MeV}$, respectively [3]. With their mixing scheme, we predict the strong decays of the two states. The results are shown in Fig. 5 It is obviously seen that the decay properties of these mixed states are not in agreement with any observations of $\Xi(1950)$.

\section{5. $J^{P}=5 / 2^{+}$assignment}

Recently, Valderrama, Xie and Nieves [22] predicted that the narrow structure with a mass of $M \simeq 1963 \mathrm{MeV}$ observed in the $\Lambda \bar{K}$ channel by Biagi et al. [21] (denoted by $\Xi(1963)$ ) could be assigned to the partner of the $J^{P}=5 / 2^{+} N(1680)$, $\Lambda(1820)$ and $\Sigma(1915)$ resonances. According to the classification of quark model, these resonances could be roughly considered as the $\left|56,{ }^{2} 8,2,2, \frac{5}{2}^{+}\right\rangle$configuration [61]. Firstly, we calculate the strong decays of the $N(1680), \Lambda(1820)$ and $\Sigma(1915)$ states, which are listed in Tab. IX. From the table, it is found that the decay properties of $N(1680), \Lambda(1820)$ and $\Sigma(1915)$ could be roughly understood by taking them as the $\left|56,{ }^{2} 8,2,2, \frac{5}{2}^{+}\right\rangle$configuration. According to the model predictions, the mass of $\Xi\left|56,{ }^{2} 8,2,2, \frac{5}{2}^{+}\right\rangle$is close to $1963 \mathrm{MeV}$ [3, 9]. Assigning $\Xi(1963)$ to the $\left|56,{ }^{2} 8,2,2, \frac{5}{2}^{+}\right\rangle$configuration, the predicted strong decay properties are shown in Tab. IX as well. It is seen that although the decay width is in agreement with the data, the partial decay width ratios are not consistent with the observations of Biagi et al. [21] at all. 
TABLE IX: The total and partial decay widths $(\mathrm{MeV})$ of the wellestablished four-star baryons $N(1680), \Sigma(1915)$ and $\Lambda(1820)$, which are considered the pure $\left|56,{ }^{2} 8,2,2, \frac{5}{2}^{+}\right\rangle$state. The data are obtained from PDG.

\begin{tabular}{|l|c|c|c|c|c|c|}
\hline \hline assignment & channel & $\Gamma_{i}^{\text {th }}$ & $\Gamma_{\text {total }}^{\text {th }}$ & $\Gamma_{\text {total }}^{\text {exp }}$ & $\left.\frac{\Gamma_{i}}{\Gamma_{\text {total }}}\right|_{\text {th }}$ & $\left.\frac{\Gamma_{i}}{\Gamma_{\text {total }}}\right|_{\text {exp }}$ \\
\hline$N(1680) \frac{5}{2}^{+}$ & $n \pi$ & 38.0 & 59 & $120 \sim 140$ & 0.64 & $0.65-0.70$ \\
& $\Delta \pi$ & 21.0 & & & 0.36 & $\ldots$ \\
& $N \eta$ & 0.4 & & & 0.01 & $0.0-0.01$ \\
& $\Lambda K$ & 0.03 & & & 0 & $\ldots$ \\
\hline$\Sigma(1915) \frac{5}{2}^{+}$ & $N \bar{K}$ & 1.2 & 63 & $80 \sim 160$ & 0.02 & $0.05-0.15$ \\
& $\Lambda \pi$ & 10.4 & & & 0.16 & $\ldots$ \\
& $\Sigma \pi$ & 26.7 & & & 0.42 & $\ldots$ \\
& $\Sigma(1385) \pi$ & 6.2 & & & 0.10 & $\ldots$ \\
& $\Delta(1232) \bar{K}$ & 18.4 & & & 0.29 & $\ldots$ \\
\hline$\Lambda(1820) \frac{5}{2}^{+}$ & $N \bar{K}$ & 14.8 & 30 & $70 \sim 90$ & 0.49 & $0.55-0.65$ \\
& $\Sigma \pi$ & 5.8 & & & 0.19 & $0.08-0.14$ \\
& $\Sigma(1385) \pi$ & 9.8 & & & 0.32 & $\ldots$ \\
\hline$\Xi(1963) \frac{5}{2}^{+}$ & $\Xi \pi$ & 1.0 & 19 & $25 \pm 15$ & 0.05 & \\
& $\Sigma \bar{K}$ & 9.2 & & & 0.48 & \\
& $\Lambda \bar{K}$ & 0.8 & & & 0.04 & \\
& $\Xi(1530) \pi$ & 5.2 & & & 0.27 & \\
& $\Sigma(1385) \bar{K}$ & 2.5 & & & 0.13 & \\
\hline
\end{tabular}

Furthermore, Chao et al. also predicted a mixed states with $J^{P}=5 / 2^{+}$having a mass of $M=1935 \mathrm{MeV}$ [3]. With their mixing scheme, its strong decays are studied. The results are shown in Fig. 5 as well. It is seen that the strong decays are dominated by the $\Sigma \bar{K}$ channel, which disagrees with any observations of $\Xi(1950)$.
In brief, there might be several $\Xi$ resonances in the $1900-$ $2000 \mathrm{MeV}$ region observed in experiments. These states are most likely to correspond to the pure octet $\Xi$ resonance with $J^{P}=5 / 2^{-}$, the mixed state $\left|\Xi \frac{1}{2}^{-}\right\rangle_{3}$ with $J^{P}=1 / 2^{-}$(its main component is $\left.\left|70,{ }^{4} 8,1,1, \frac{1}{2}^{-}\right\rangle\right)$, the second orbital excitation $\left|56,{ }^{4} 10,2,2, \frac{1}{2}^{+}\right\rangle$with $J^{P}=1 / 2^{+}$, etc. These states can be easily distinguished by the measurements of their partial decay width ratios, $\Gamma(\Lambda \bar{K}) / \Gamma(\Sigma \bar{K})$ and $\Gamma(\Sigma \bar{K}) / \Gamma(\Xi \pi)$.

TABLE X: The decay widths (MeV) of $\Xi(2030)$ with different assignments.

\begin{tabular}{|c|c|c|c|c|c|c|c|c|c|}
\hline \hline assignment & $\Xi \pi$ & $\Sigma \bar{K}$ & $\Lambda \bar{K}$ & $\Xi(1530) \pi$ & $\Sigma(1385) \bar{K}$ & $\Lambda(1405) \bar{K}$ & $\Lambda(1520) \bar{K}$ & $\Gamma_{\text {total }}$ & $\Gamma(\Lambda \bar{K}): \Gamma(\Sigma \bar{K})$ \\
\hline$\left|56,{ }^{2} 8,2,2, \frac{5}{2}^{+}\right\rangle$ & 1.6 & 18.5 & 1.3 & 8.2 & 5.7 & 0.4 & 0.01 & 35.8 & 0.07 \\
\hline$\left|70,{ }^{2} 8,2,2, \frac{3}{2}^{+}\right\rangle$ & 1.4 & 17.9 & 4.5 & 1.4 & 0.6 & 1.5 & 5.4 & 32.7 & 0.25 \\
$\left|70,{ }^{2} 8,2,2, \frac{5}{2}^{+}\right\rangle$ & 0.8 & 5.8 & 2.7 & 4.1 & 2.8 & 0.05 & 0.07 & 16.3 & 0.46 \\
\hline
\end{tabular}

\section{E. $\Xi(2030)$}

$\Xi(2030)$ is a three-star state listed in PDG. It mainly decays into $\Sigma \bar{K}$ and $\Lambda \bar{K}$ channels. The decay ratios into the other channels, such as $\Xi \pi$ and $\Xi(1530) \pi$, are small. The measured decay width and partial decay width ratio are

$$
\Gamma \simeq 21 \pm 6 \mathrm{MeV}, \frac{\Gamma(\Lambda \bar{K})}{\Gamma(\Sigma \bar{K})} \simeq 0.22 \pm 0.09
$$

A moment analysis of the HEMINGWAY 77 data indicated at a level of three standard deviations that $J \geq 5 / 2$ [23].

We analyze the strong decay properties for all the configurations in the $N=2$ shell, which are shown in Figs. 3,7 From the figures, we find that only three excitations $\left|56,{ }^{2} 8,2,2, \frac{5}{2}^{+}\right\rangle$, $\left|70,{ }^{2} 8,2,2, \frac{3}{2}^{+}\right\rangle$and $\left|70,{ }^{2} 8,2,2, \frac{5}{2}^{+}\right\rangle$have comparable decay widths with that of $\Xi(2030)$, and mainly decay into $\Sigma \bar{K}$ channel. Considering them as assignments to $\Xi(2030)$, we collect their decay properties in Tab. $\mathrm{X}$, where we find that both the decay width and the partial decay ratio

$$
\Gamma \simeq 33 \mathrm{MeV}, \frac{\Gamma(\Lambda \bar{K})}{\Gamma(\Sigma \bar{K})} \simeq 0.25
$$

of the $\left|70,{ }^{2} 8,2,2, \frac{3}{2}^{+}\right\rangle$configuration are in good agreement with the observations. However, its spin $J=3 / 2$ disagrees with the moment analysis of the HEMINGWAY 77 data [23].

As a whole, if we do not care about the moment analysis of the HEMINGWAY 77 data [23], $\Xi(2030)$ favors the $\left.170,{ }^{2} 8,2,2, \frac{3}{2}^{+}\right\rangle$assignment. $\Xi(2030)$ could not be assigned to any pure $J^{P}=7 / 2^{+}$states or any admixtures between them. If the spin-parity of $\Xi(2030)$ is $J^{P}=5 / 2^{+}$, it is most likely to be a mixed state, for no pure $J^{P}=5 / 2^{+}$configuration could explain the data. 


\section{SUMMARY}

In this work, we have studied the strong decays of the $\Xi$ baryons within $N \leq 2$ shells in a chiral quark model. The strong decay properties of these well-established ground decuplet baryons could be reasonably described. We find that $\Xi(1690)$ should be assigned to the spin-parity $J^{P}=1 / 2^{-}$ state $\left|70,{ }^{2} 8,1,1, \frac{1}{2}^{-}\right\rangle$, which might slightly mix with the other configurations. The $J^{P}=1 / 2^{-}$for $\Xi(1690)$ predicted by us is consistent with the suggestions from the constituent quark model [11], Skyrme model [12] and unitary chiral approaches [15, 16]. The strong decays of the physical partners of $\Xi(1690),\left|\Xi \frac{1}{2}^{-}\right\rangle_{1}$ and $\left|\Xi \frac{1}{2}^{-}\right\rangle_{3}$ are analyzed as well. $\left|\Xi \frac{1}{2}^{-}\right\rangle_{1}$ might be observed in the $\Sigma \bar{K}$ and $\Lambda \bar{K}$ channels, while $\left|\Xi \frac{1}{2}^{-}\right\rangle_{3}$ is possibly observed in the $\Xi \pi$ and $\Lambda \bar{K}$ channels.

The strong decay properties of $\Xi(1820)$ could be well understood by assigning it to $\left|70,{ }^{2} 8,1,1, \frac{3}{2}^{-}\right\rangle$. There might exist slight configuration mixing in $\Xi(1820)$. Its main component is $\left|70,{ }^{2} 8,1,1, \frac{3}{2}^{-}\right\rangle(\sim 92 \%)$, which is compatible with the predictions of the quark model [3], and large $N_{c}$ QCD approach [7, 8]. Considering the configuration mixing effects, we also have studied the strong decay properties of the physical partners of $\Xi(1820),\left|\Xi \frac{3}{2}^{-}\right\rangle_{1}$ and $\left|\Xi \frac{3}{2}^{-}\right\rangle_{3}$. The observations in the $\Sigma(1385) \bar{K}$ and $\Xi(1530) \pi$ channels are crucial to look for the other $J^{P}=3 / 2^{-}$states $\left|\Xi \frac{3}{2}^{-}\right\rangle_{1}$ and $\left|\Xi \frac{3}{2}^{-}\right\rangle_{3}$ in future experiments.

The situation for $\Xi(1950)$ is very complicated. Several $\Xi$ resonances in the $1900-2000 \mathrm{MeV}$ region might have been observed in experiments, which is supported by the mass calculations from various models [3, 7-9], and the recent strong decay analysis in [22]. The broad $\Xi$ resonances observed in the $\Xi \pi$ channel might be good candidates for the $J^{P}=5 / 2^{-}$octet state or the mixed state $\left|\Xi \frac{1}{2}^{-}\right\rangle_{3}$ with $J^{P}=1 / 2^{-}$. The $\Xi$ resonance with moderate width ob- served by Goldwasser et al. [60] might correspond to the $J^{P}=1 / 2^{+}$excitation $\left|56,{ }^{4} 10,2,2, \frac{1}{2}^{+}\right\rangle$. The existence of a $J^{P}=1 / 2^{+} \Xi$ excitation with a mass around $1950 \mathrm{MeV}$ is also suggested by $\mathrm{Oh}$ in a Skyrme model [12]. The second orbital excitation $\left|56,{ }^{4} 10,2,2, \frac{3}{2}^{+}\right\rangle$and the mixed state $\left|\Xi \frac{1}{2}^{-}\right\rangle_{1}$ might be candidates for a narrow width state observed in the $\Lambda \bar{K}$ channel; however, their spin-parity is not consistent with a moment analysis of the data. The partial decay ratios, $\Gamma(\Lambda \bar{K}) / \Gamma(\Sigma \bar{K})$ and $\Gamma(\Sigma \bar{K}) / \Gamma(\Xi \pi)$, are sensitive to different assignments. Thus, the measurements of these ratios are crucial to uncover many puzzles in $\Xi(1950)$.

In present work, $\Xi(2030)$ as any spin-parity $J^{P}=7 / 2^{+}$ states should be excluded. The observations of $\Xi(2030)$ do not favor any pure $J^{P}=5 / 2^{+}$configuration as well. If we do not care about the moment analysis of the HEMINGWAY 77 data, $\Xi(2030)$ favors the $\left|70,{ }^{2} 8,2,2, \frac{3}{2}^{+}\right\rangle$assignment. Further observations in the $\Xi(1530) \pi$ and $\Sigma(1385) \bar{K}$ channels are necessary.

To provide helpful information for the search for the missing $\Xi$ baryons, in Figs. 3] 7 our predictions of their strong decay properties are shown as well. From our theoretical results, we find that the strong decays of many $\Xi$ resonances are dominated by the $\Xi(1530) \pi$ and $\Sigma(1385) \bar{K}$, thus, in these decay channels, we might find some new $\Xi$ resonances as well.

\section{Acknowledgements}

This work is supported, in part, by the National Natural Science Foundation of China (Grant No. 11075051), Program for Changjiang Scholars and Innovative Research Team in University (Grant No. IRT0964), the Program Excellent Talent Hunan Normal University, and the Hunan Provincial Natural Science Foundation (Grants No.11JJ7001 and No. 13JJ1018).
[1] J. Beringer et al. [Particle Data Group Collaboration], Phys. Rev. D 86, 010001 (2012).

[2] N. Isgur and G. Karl, Phys. Rev. D 18, 4187 (1978).

[3] K. -T. Chao, N. Isgur and G. Karl, Phys. Rev. D 23, 155 (1981).

[4] S. Capstick and N. Isgur, Phys. Rev. D 34, 2809 (1986).

[5] L. Y. .Glozman and D. O. Riska, Phys. Rep. 268, 263 (1996) [hep-ph/9505422].

[6] R. Bijker, F. Iachello and A. Leviatan, Ann. Phys. 284, 89 (2000) |nucl-th/0004034|.

[7] C. L. Schat, J. L. Goity and N. N. Scoccola, Phys. Rev. Lett. 88, 102002 (2002) |hep-ph/0111082|.

[8] J. L. Goity, C. L. Schat and N. N. Scoccola, Phys. Rev. D 66, 114014 (2002) |hep-ph/0209174|.

[9] J. L. Goity, C. Schat and N. N. Scoccola, Phys. Lett. B 564, 83 (2003) |hep-ph/0304167|.

[10] F. X. Lee and X. Y. Liu, Phys. Rev. D 66, 014014 (2002) [nucl-th/0203051].

[11] M. Pervin and W. Roberts, Phys. Rev. C 77, 025202 (2008) [arXiv:0709.4000 [nucl-th]].

[12] Y. Oh, Phys. Rev. D 75, 074002 (2007) [hep-ph/0702126] [hep$\mathrm{ph}]$.
[13] T. Melde, W. Plessas and B. Sengl, Phys. Rev. D 77, 114002 (2008) arXiv:0806.1454 [hep-ph]].

[14] Y. Chen and B. -Q. Ma, Nucl. Phys. A 831, 1 (2009) arXiv:0911.1156 [hep-ph]].

[15] E. E. Kolomeitsev and M. F. M. Lutz, Phys. Lett. B 585, 243 (2004) |nucl-th/0305101].

[16] S. Sarkar, E. Oset and M. J. Vicente Vacas, Nucl. Phys. A 750, 294 (2005) [Erratum-ibid. A 780, 78 (2006)] nucl-th/0407025|.

[17] B. Aubert et al. [BABAR Collaboration], Phys. Rev. D 78, 034008 (2008) [arXiv:0803.1863 [hep-ex]].

[18] D. Faiman and D. E. Plane, Nucl. Phys. B 50, 379 (1972).

[19] N. Matagne and F. .Stancu, Phys. Rev. D 83, 056007 (2011) arXiv:1101.5304 [hep-ph]].

[20] J. Alitti, E. Flaminio, W. Metzger, D. Radojicic, R. R. Rau, N. P. Samios, I. Skillicorn and C. R. Richardson et al., Phys. Rev. Lett. 21, 1119 (1968).

[21] S. F. Biagi, M. Bourquin, R. M. Brown, H. J. Burckhart, P. Extermann, M. Gailloud, C. N. P. Gee and W. M. Gibson et al.,Z. Phys. C 34, 175 (1987).

[22] M. Pavon Valderrama, J. -J. Xie and J. Nieves, Phys. Rev. D 85, 
017502 (2012) arXiv:1111.2218 [hep-ph]].

[23] R. J. Hemingway et al. [Amsterdam-CERN-Nijmegen-Oxford Collaboration], Phys. Lett. B 68, 197 (1977).

[24] N. P. Samios, M. Goldberg and B. T. Meadows, Rev. Mod. Phys. 46, 49 (1974).

[25] T. Nagae, Nucl. Phys. A 805, 486 (2008).

[26] M. F. M. Lutz et al. [PANDA Collaboration], arXiv:0903.3905 [hep-ex].

[27] J. W. Price et al. [CLAS Collaboration], Phys. Rev. C 71, 058201 (2005) |nucl-ex/0409030|.

[28] J. W. Price et al. [CLAS Collaboration], Nucl. Phys. A 754, 272 (2005) [nucl-ex/0402006|.

[29] L. Guo, D. P. Weygand, M. Battaglieri, R. D. Vita, V. Kubarovsky, P. Stoler, M. J. Amaryan and P. Ambrozewicz et al., Phys. Rev. C 76, 025208 (2007) [nucl-ex/0702027].

[30] B. -S. Zou, Nucl. Phys. A 684, 330 (2001) [hep-ph/0006039].

[31] D. M. Asner, T. Barnes, J. M. Bian, I. I. Bigi, N. Brambilla, I. R. Boyko, V. Bytev and K. T. Chao et al., Int. J. Mod. Phys. A 24, S1 (2009) [arXiv:0809.1869 [hep-ex]].

[32] D. A. Sharov, V. L. Korotkikh and D. E. Lanskoy, Eur. Phys. J. A 47, 109 (2011) [arXiv:1105.0764 [nucl-th]].

[33] R. Shyam, O. Scholten and A. W. Thomas, Phys. Rev. C 84, 042201 (2011) [arXiv:1108.2318 [hep-ph]].

[34] J. K. S. Man, Y. Oh and K. Nakayama, Phys. Rev. C 83, 055201 (2011) |arXiv:1103.1699 [nucl-th]].

[35] K. Nakayama, Y. Oh and H. Haberzettl, Phys. Rev. C 74, 035205 (2006) |hep-ph/0605169|.

[36] K. Nakayama, Y. Oh and H. Haberzettl, Phys. Rev. C 85, 042201 (2012) |arXiv:1201.5598 [hep-ph]].

[37] X. H. Zhong and Q. Zhao, Phys. Rev. D 77, 074008 (2008) [arXiv:0711.4645 [hep-ph]].

[38] L. -H. Liu, L. -Y. Xiao and X. -H. Zhong, Phys. Rev. D 86, 034024 (2012) [arXiv:1205.2943 [hep-ph]].

[39] X. H. Zhong and Q. Zhao, Phys. Rev. D 78, 014029 (2008) [arXiv:0803.2102 [hep-ph]].

[40] X. H. Zhong, Phys. Rev. D 82, 114014 (2010) [arXiv:1009.0359 [hep-ph]].

[41] X. H. Zhong and Q. Zhao, Phys. Rev. D 81, 014031 (2010) [arXiv:0911.1856 [hep-ph]].
[42] Rajat K.Bhaduri, Models of the Nucleon: From Quarks to Soliton (Addison-Wesley, Reading, MA, 1988).

[43] G. Karl and E. Obryk, Nucl. Phys. B 8, 609 (1968).

[44] Zhenping Li, Ph.D. thesis, University of Tennessee, 1991.

[45] L. A. Copley, N. Isgur and G. Karl, Phys. Rev. D 20, 768 (1979) [Erratum-ibid. D 23, 817 (1981)].

[46] R. Koniuk and N. Isgur, Phys. Rev. D 21, 1868 (1980) [Erratumibid. D 23, 818 (1981)].

[47] Z. P. Li, Phys. Rev. C 52, 1648 (1995) [hep-ph/9502218].

[48] Z. P. Li, H. X. Ye and M. H. Lu, Phys. Rev. C 56, 1099 (1997) [arXiv:nucl-th/9706010].

[49] Q. Zhao, J. S. Al-Khalili, Z. P. Li and R. L. Workman, Phys. Rev. C 65, 065204 (2002) [arXiv:nucl-th/0202067].

[50] X. H. Zhong, Q. Zhao, J. He and B. Saghai, arXiv:0706.3543 [nucl-th].

[51] X. H. Zhong and Q. Zhao, Phys. Rev. C 84, 065204 (2011) |arXiv:1110.5466 [nucl-th]].

[52] C. S. An and B. Saghai, Phys. Rev. C 84, 045204 (2011) |arXiv:1108.3282 [nucl-th]].

[53] C. Dionisi et al. [Amsterdam-CERN-Nijmegen-Oxford Collaboration], Phys. Lett. B 80, 145 (1978).

[54] B. Aubert et al. [BABAR Collaboration], hep-ex/0607043

[55] J. Alitti, V. E. Barnes, E. Flaminio, W. Metzger, D. Radojicic, R. R. Rau, C. R. Richardson and N. P. Samios et al., Phys. Rev. Lett. 22, 79 (1969).

[56] S. Apsell, N. Barash-Schmidt, L. Kirsch, P. Schmidt, C. Y. Chang, R. J. Hemingway, B. V. Khoury and A. R. Stottlemyer et al., Phys. Rev. Lett. 24, 777 (1970).

[57] J. Badier, M. Demoulin and J. Goldberg, Phys. Lett. 16, 171 (1965).

[58] E. Briefel, S. A. Gourevitch, L. Kirsch, P. Schmidt, C. Y. Chang, R. J. Hemingway, B. V. Khoury and A. R. Stottlemyer et al., Phys. Rev. D 16, 2706 (1977).

[59] V. Guzey and M. V. Polyakov, hep-ph/0512355

[60] E. L. Goldwasser and P. F. Schultz, Phys. Rev. D 1, 1960 (1970).

[61] E. Klempt and J. M. Richard, Rev. Mod. Phys. 82, 1095 (2010) arXiv:0901.2055 [hep-ph]]. 